\title{
A Bayesian Method for Water Resources Vulnerability Assessment: A Case Study of the Zhangjiakou Region, North China
}

\author{
Xuan Wang, ${ }^{1,2}$ Fangbing Ma, ${ }^{1,3}$ Chunhui Li, ${ }^{1,2}$ and Jie Zhu ${ }^{1,2}$ \\ ${ }^{1}$ State Key Laboratory of Water Environment Simulation, School of Environment, Beijing Normal University, Beijing 100875, China \\ ${ }^{2}$ Key Laboratory of Water and Sediment Sciences of Ministry of Education, School of Environment, Beijing Normal University, \\ Beijing 100875, China \\ ${ }^{3}$ Shidu Town People's Government, Beijing 102411, China
}

Correspondence should be addressed to Xuan Wang; wangx@bnu.edu.cn

Received 18 June 2014; Accepted 23 August 2014

Academic Editor: Jian Li

Copyright (C) 2015 Xuan Wang et al. This is an open access article distributed under the Creative Commons Attribution License, which permits unrestricted use, distribution, and reproduction in any medium, provided the original work is properly cited.

\begin{abstract}
Water resources vulnerability (WRV) assessment is an important basis for maintaining water resources security in a basin. In this paper, considering the complexity of the water resources system and the uncertainty of the assessment information, a method based on the Bayesian theory was developed for performing WRV assessments while using the constructed indicator system. This system includes four subsystems, the hydrological subsystem, the socioeconomic subsystem, the ecoenvironmental subsystem and the hydraulic engineering subsystem. The WRV degree for each subsystem and the integrated water resources system were assessed. Finally, the assessment results and the characteristics of the Bayesian method were compared with those of the grey relational analysis method and the parametric-system method. The results showed the following. (1) The WRV of the integrated water resources system of the entire Zhangjiakou region was very high; Zhangjiakou City and Xuanhua County have tendencies to belong to Extreme WRV, with probabilities of $26.8 \%$ and $25 \%$, respectively, while the other seven administrative counties have tendencies to belong to High WRV, with probabilities ranging from $24.6 \%$ to $27 \%$. (2) Compared with the parametric-system method and the grey relational analysis method, the Bayesian method is simple and can effectively address the uncertainty issues with the reliable WRV assessment results.
\end{abstract}

\section{Introduction}

Under the influence of climate change and human activities, many countries in the world are faced with water problems, such as water shortages, water pollution and frequent occurrences of extreme hydrological events, which have put pressure on water availability $[1,2]$. Thus, it is of importance to study the recovery capability of a water resources system after being disturbed. Besides reliability, resilience, and sustainability, vulnerability is an important indicator to evaluate the performance of a water resources system [3-5]. Water resources vulnerability (WRV) can be defined as the ease with which a water resources system can be threatened and damaged by both natural disasters and human activities. Once this damage occurs, it is difficult for the system to recover to its former status. WRV is generally reflected by such factors as the quantity and quality of the surface water and groundwater and the carrying capacity of the water resources [6]. The purpose of WRV assessment is to understand the water resources status in a certain region, to find the main influencing factors of the $\mathrm{WRV}$, and then to propose appropriate countermeasures to reduce the WRV. In recent years, following the continuous development of economic and social progress, the growth of the population as well as the development of the urbanization and industrialization, the demand for water resources is increasing, and the supply is tending toward shortages. At the same time, excessive production activities lead to a decline in water's ecological value. Having addressed water security issues that are associated with WRV and provided references 
for the protection, rational development and utilization of regional water resources, WRV assessment has been drawing more attention.

Since the late 1960s, many scholars and water managers around the world have studied the WRV, but most of them concentrated on groundwater vulnerability assessment [710]. With respect to surface water vulnerability, most of the literature focused on the supply and demand balance of water resources or selected several indicators and methods to evaluate the carrying capacity or sustainability of the water resources [11]. Given the uneven distribution of water resources in space and time, it is necessary to pay close attention to the WRV that is caused by climate change and human activities. It is also important to enhance the joint assessment of surface water and groundwater, water quantity, and water quality, to ensure a sustainable utilization of water resources of a given region. In recent years, many approaches have been developed to assess WRV, such as overlay and indicator methods [12-14], process-based simulation methods $[15,16]$, statistical methods $[9,17]$, and fuzzy comprehensive judgment methods [18]. For obtaining more reliable assessment results, these methods required a large amount of natural environmental and socioeconomic data with high accuracy. In [19], Li quantitatively analyzed the regional WRV by using the fuzzy optimization (FO) method, the grey relational analysis (GRA) method, and the parametric-system (PS) method by constructing an indicator system with a comprehensive consideration of surface water and groundwater, water quantity, and water quality. These methods, however, cannot address the uncertainty that is inherent in all of the vulnerability assessments [20]. With the increase in the pressure exerted by socioeconomic development on a natural environment, the complexity and uncertain characteristics of WRV that arise from both climate change and human activities has become even more obvious. To improve the water resources management and protection, the regional WRV research that considers fuzziness and uncertainty of the assessment results is highly desired.

With increasingly in-depth research on uncertain events, the Bayesian method has received extensive attention [21]. It uses objective probability estimations and incomplete information to estimate the probability of some unknown states. This method can also describe uncertain information flexibly and conduct uncertainty reasoning [22]. Compared with the existing assessment methods, the Bayesian method's superiority lies in giving the probability or tendency to a certain grade to which the system belongs, not just simply giving the grade. In addition, it has the advantages of a simple principle and calculation process and can obtain reliable results for small samples. Thus so far, it has been widely used for water quality assessment [23-25], fault diagnosis, and risk assessment [26, 27], but it has rarely been used for WRV assessment. The application of the Bayesian method in actual WRV assessment is expected for the effective management of regional water resources.

The objectives of this research were to (a) develop a Bayesian method for WRV assessment; (b) apply the proposed method to assess the WRV condition of the nine counties of the Zhangjiakou region, and describe the probability distribution characteristics at different counties; and (c) compare the results with the existing methods, including the GRA method and the PS method. The proposed method will improve the existing method in addressing uncertain information, and the assessment results can help to identify specific water resources problems of the Guanting Reservoir, in such a way that appropriate measures can be taken for effective environmental management.

\section{Study Area}

2.1. Overview of the Study Area. The Zhangjiakou region, which is located in the upper reaches of the Guanting Reservoir Basin, is selected as a case to assess the regional WRV (Figure 1). It is confronted with the dual task of developing the economy and protecting the natural ecological environment. The concerned city and counties are Zhangjiakou City, Huai'an County, Wanquan County, Xuanhua County, Yuxian County, Yangyuan County, Zhuolu County, Huailai County, and Chongli County, with 17.8 thousand $\mathrm{km}^{2}$ accounting for $40.5 \%$ of the area of Guanting Reservoir Basin. The Zhangjiakou region has a continental monsoon climate, with an annual mean temperature ranging from $7^{\circ} \mathrm{C}$ to $10^{\circ} \mathrm{C}$. The annual mean precipitation is rather low (approximately $400 \mathrm{~mm}$ ), and $80 \%$ of the rainfall occurs between June and September. The topography in the Zhangjiakou region is very complex; mountains, hills, and rivers account for approximately $30 \%$ of the area, respectively. The population of the region is 4.59 million, and the water resources per capita are $399 \mathrm{~m}^{3}$, which is less than the internationally accepted standard of extreme water scarcity of $500 \mathrm{~m}^{3}$ [28].

The region has a backward economic development. Excessive consumption and pollution of water resources have exerted an enormous pressure on local water resources system and have directly influenced the security of the water supply of Beijing [29]. Therefore, an effective WRV assessment is necessary and desired for regional water resources protection and management. The hydrological data came from Zhangjiakou Water Conservancy Bureau, the water quality data came from Environmental Protection Agency of Zhangjiakou, and the ecoenvironmental and socioeconomic data were from the statistical yearbooks of the Zhangjiakou region [19].

2.2. Key Factors Influencing WRV. Regional WRV is reflected by the carrying capacity, recovery capacity, and sustainability of the water resources $[6,30,31]$. The key factors that influence the WRV of the Zhangjiakou region include the following aspects.

(1) Hydrology. Influenced by the continental monsoon climate of the arid and semiarid area, the evaporation and runoff of this region have the characteristic of uneven distribution at a spatial and temporal scale. The amount of water available is not enough. The influencing factors that are associated with these hydrological aspects mainly include the natural conditions of the regional water resources, such as the 


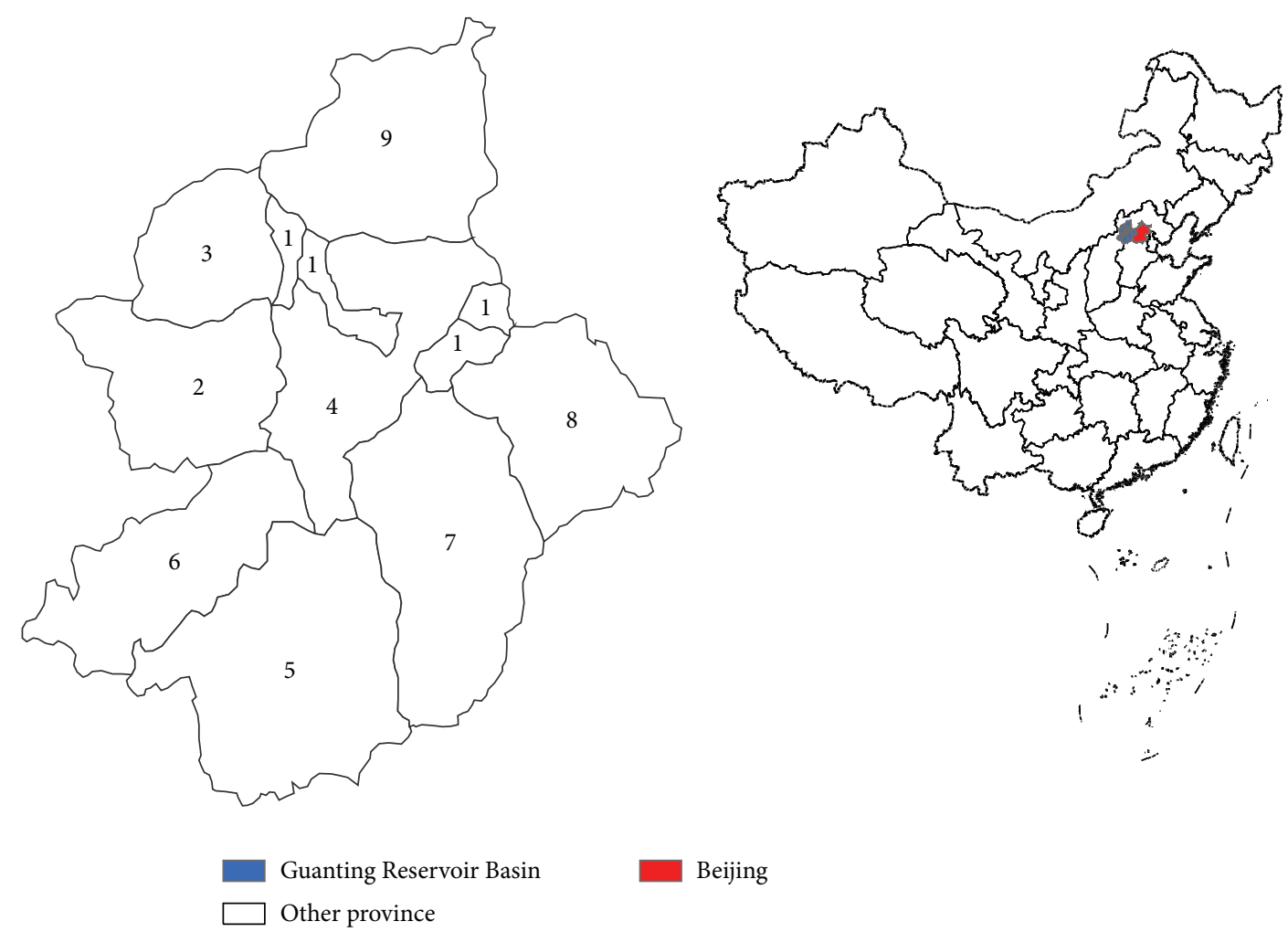

Figure 1: Location of the Zhangjiakou region, North China (1. Zhangjiakou City; 2. Huaian County; 3. Wanquan County; 4. Xuanhua County; 5. Yuxian County; 6. Yangyuan County; 7. Zhuolu County; 8. Huailai County; 9. Chongli County).

stability of the water resources system, the water abundance, the ability to renew the underground water, and the regional climate conditions.

(2) Socioeconomy. With economic development and population increase, the water resources per capita $\left(399 \mathrm{~m}^{3}\right)$ of the Zhangjiakou are much lower than the national average $\left(2100 \mathrm{~m}^{3}\right)$; thus, the region is suffering from serious water shortage issues. At the same time, the economy of Hebei Province is backward, as well as its science and technology. Extensive pattern of economic growth leads to a low utilization rate of the water resources and the increasing demand for water resources to meet the requirements of both human life and industrial production, which has imposed great pressure on the water resources. The influencing factors that are associated with the socioeconomic aspects mainly include the population pressure on the water resources, the water management level, the water supply and water demand status, the water exploitation and utilization conditions, and the water reuse rate.

(3) Ecoenvironment. The area lies in a fragile belt in terms of the ecoenvironment. The agricultural application of pesticides is approximately 2000 tons a year, and fertilizer is approximately 60,000 tons a year, which leads to serious agricultural nonpoint source pollution of the region. Since the late 1980s, rapid development of industrial and mining enterprises together with low treatment of effluent sewages has brought severe industrial point source pollution to the region. At the same time, because of the low coverage of the vegetation, the water losses and soil erosion here are very serious and the ecological environment is vulnerable. The influencing factors that are associated with the ecoenvironment mainly include the impact of land exploitation and utilization on water resources, potential pollution intensity of agricultural activities on water quality of regional surface water, and groundwater, water loss, and soil erosion.

(4) Hydraulic Engineering and Technical Management. A large number of water conservancy systems have been constructed in this region since the late 1950s, including water storage structures, abstraction systems, and other water supply projects. Hydraulic engineering can regulate and allocate water resources and prevent flood disasters, to meet the needs of the water resources of the people and the production. Thus, making use of hydraulic engineering can enhance the antiinterference ability of the water resources system. In addition, considering that agriculture is the largest supporting industry of this region, it is necessary to increase the water-saving irrigation area and improve the water use efficiency through technological advances and consciousness enhancement, to reduce its influence on WRV. The influencing factors that are associated with the hydraulic engineering and technical management mainly include the agricultural water-saving capability, as well as the regulation and storage capacity of the reservoirs. 
TABLE 1: Assessment standard of the indicators for different WRV types (Li, 2007) [19].

\begin{tabular}{|c|c|c|c|c|c|c|c|}
\hline Subsystem & Indicator & Description/reflection & $\begin{array}{c}\text { No } \\
\text { WRV }\end{array}$ & $\begin{array}{l}\text { Low } \\
\text { WRV }\end{array}$ & $\begin{array}{l}\text { Medium } \\
\text { WRV }\end{array}$ & $\begin{array}{l}\text { High } \\
\text { WRV }\end{array}$ & $\begin{array}{c}\text { Extreme } \\
\text { WRV }\end{array}$ \\
\hline \multirow{5}{*}{ A } & $\mathrm{a}_{1}$ & Regional water resources income & 1500 & 1200 & 800 & 400 & 100 \\
\hline & $a_{2}$ & System stability & 10 & 20 & 30 & 40 & 50 \\
\hline & $\mathrm{a}_{3}$ & Water abundance & 40 & 30 & 20 & 10 & 5 \\
\hline & $\mathrm{a}_{4}$ & Ground water recharge capacity & 80 & 50 & 20 & 10 & 5 \\
\hline & $a_{5}$ & Regional climate & 0.5 & 1 & 3 & 5 & 7 \\
\hline \multirow{7}{*}{$\mathrm{B}$} & $\mathrm{b}_{1}$ & Population pressure on water resources & 2500 & 2000 & 1500 & 1000 & 500 \\
\hline & $b_{2}$ & Water utilization efficiency & 100 & 350 & 600 & 850 & 1100 \\
\hline & $\mathrm{b}_{3}$ & Water utilization level & 100 & 300 & 500 & 700 & 900 \\
\hline & $\mathrm{b}_{4}$ & Water manage level & 5 & 15 & 25 & 35 & 45 \\
\hline & $b_{5}$ & Water supply and demand balance & 1 & 0.9 & 0.8 & 0.7 & 0.6 \\
\hline & $\mathrm{b}_{6}$ & Status of water resources development & 10 & 30 & 50 & 70 & 90 \\
\hline & $\mathrm{b}_{7}$ & Repetitive utilization degree of industrial water & 80 & 65 & 50 & 35 & 20 \\
\hline \multirow{6}{*}{$\mathrm{C}$} & $\mathrm{c}_{1}$ & $\begin{array}{l}\text { Impacts of land development on water } \\
\text { resources }\end{array}$ & 30 & 350 & 65 & 80 & 90 \\
\hline & $c_{2}$ & $\begin{array}{l}\text { Agricultural pollution intensity on water } \\
\text { quality }\end{array}$ & 10 & 25 & 280 & 1000 & 1500 \\
\hline & $c_{3}$ & Surface water quality & 0 & 0.1 & 0.2 & 0.5 & 1 \\
\hline & $\mathrm{c}_{4}$ & Groundwater quality & 0 & 5 & 35 & 60 & 85 \\
\hline & $c_{5}$ & Area changes of soil and water loss & 0 & 10 & 20 & 30 & 40 \\
\hline & $\mathrm{c}_{6}$ & Degree of sewage disposal & 90 & 75 & 60 & 45 & 30 \\
\hline \multirow{3}{*}{$\mathrm{D}$} & $\mathrm{d}_{1}$ & Water saving capacity of agriculture & 80 & 60 & 40 & 30 & 20 \\
\hline & $\mathrm{d}_{2}$ & Regulating capacity of hydraulic engineering & 50 & 40 & 30 & 20 & 10 \\
\hline & $\mathrm{d}_{3}$ & Storage and release capacity of reservoirs & 80 & 65 & 50 & 40 & 30 \\
\hline
\end{tabular}

Note: A: hydrological subsystem; B: socioeconomic subsystem; C: ecoenvironmental subsystem; D: hydraulic engineering subsystem; $a_{1}$ : mean annual precipitation $(\mathrm{mm}) ; \mathrm{a}_{2}$ : annual relative variability of precipitation $(\%) ; \mathrm{a}_{3}$ : mean water resources amount of unit area $\left(10^{4} \mathrm{~m}^{3} / \mathrm{km}^{2}\right) ; \mathrm{a}_{4}$ : groundwater recharge of unit area $\left(10^{4} \mathrm{~m}^{3} / \mathrm{km}^{2}\right) ; a_{5}$ : drought index; $b_{1}$ : water resources per capita $\left(\mathrm{m}^{3} /\right.$ capita); $b_{2}$ : water consumption per unit GDP $\left(\mathrm{m}^{3} / 10^{4}\right.$ yuan); $b_{3}:$ water consumption per capita $\left(\mathrm{m}^{3} /\right.$ capita); $\mathrm{b}_{4}$ : difference between planned and actual water utilization amount $(\%) ; b_{5}$ : water supply and demand ratio; $b_{6}$ : water resources development and utilization ratio (\%); $b_{7}$ : repetitive utilization ratio of industrial water (\%); $c_{1}$ : land development ratio (\%); $c_{2}$ : fertilizer use intensity $(\mathrm{kg} / \mathrm{ha}) ; \mathrm{c}_{3}$ : pollution index of surface water; $\mathrm{c}_{4}$ : ratio of polluted groundwater exceeding the standard (\%); $\mathrm{c}_{5}$ : ratio of soil and water loss (\%); $\mathrm{c}_{6}$ : disposal ratio of sewage (\%); $d_{1}$ : water-saving irrigation ratio $(\%) ; d_{2}$ : ratio of design utilizable storage of reservoir to surface water quantity $(\%) ; d_{3}:$ ratio of effective storage to total storage of reservoirs (\%).

The four aspects interact with each other, for example, the increase in the population and water demand and the decrease in the water resources per capita can make the water shortage even more serious. At the same time, the increase in the pollutant emissions leads to a significant deterioration in the water environment. Over disafforestation, farming, and husbandry lead to a low coverage of the vegetation, thus aggravating the water losses and soil erosion. The disharmonious development of the natural resources and environment has reduced the carrying capacity, the recovery capacity, and the sustainability of the water resources system and increased the WRV. Therefore, it is necessary to consider the above four aspects comprehensively, to establish an indicator system that considers the supply and demand balance of the water resources and that combines the water quantity with the water quality, to fully reflect the influence of the climate change and human activities.

\section{Methodology}

3.1. Conceptual Model Diagram for WRV Assessment Based on Bayesian Theory. In [19], Li divided WRV into four subsystems, the hydrological subsystem, the socioeconomic subsystem, the ecoenvironmental subsystem, and the hydraulic engineering subsystem, and then constructed an indicator system for the WRV assessment. The vulnerable degree of every indicator was divided into five levels, which were No WRV, Low WRV, Medium WRV, High WRV, and Extreme WRV (see Table 1).

Based on Bayesian theory, we calculated the posterior probability of every indicator, and then, we calculated the posterior probability of the multi-indicator comprehensive WRV assessment of each subsystem. For the multilayered WRV assessment, the subsystem in one layer can be seen as an indicator of the next layer; then, we recalculated the posterior 


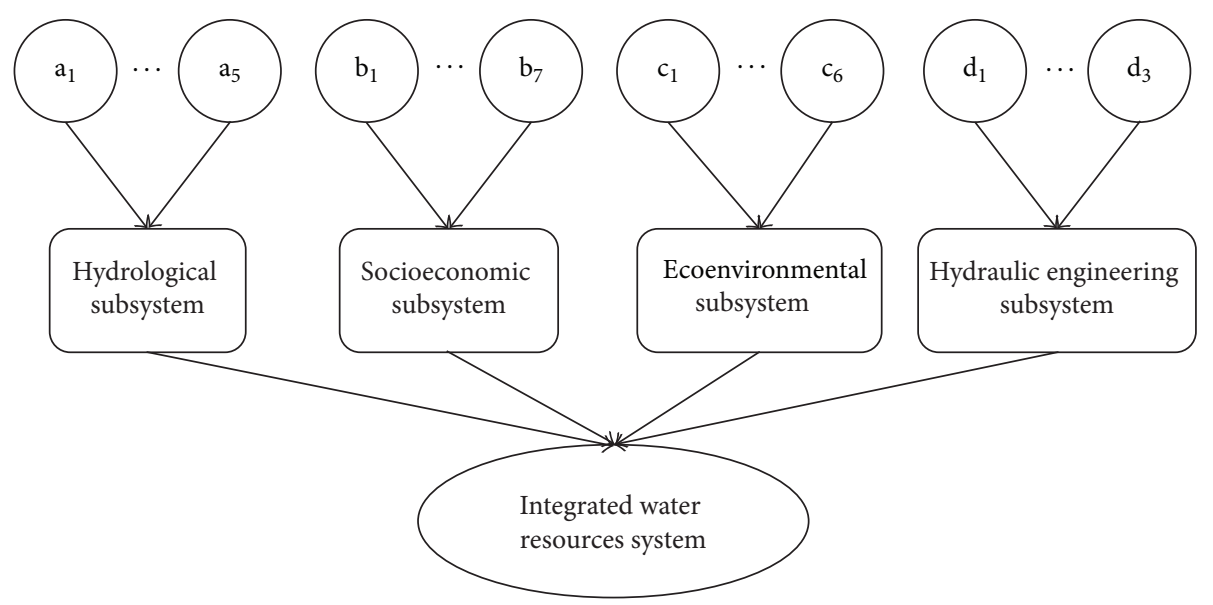

FIGURE 2: The relationship among the indicators, subsystems, and integrated system.

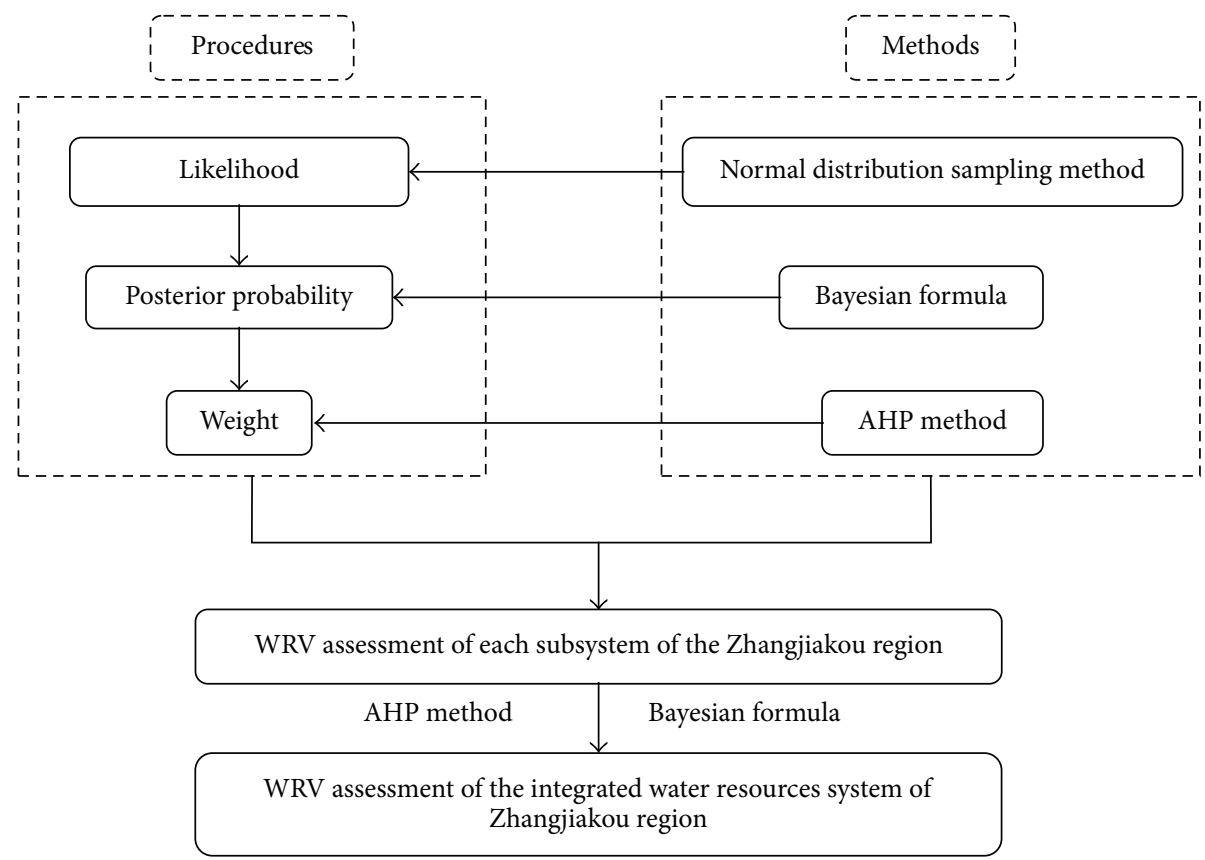

FIGURE 3: Procedures for WRV assessment by using the Bayesian method.

probability of the multisubsystem WRV assessment of the integrated water resources system with the Bayesian formula (Figure 2).

3.2. Procedures for the WRV Assessment Model Based on the Bayesian Theory. We used the Bayesian formula [32] to calculate the probability of a system belonging to a certain WRV type, to identify the vulnerable degree of the water resources system. The WRV assessment procedures with the Bayesian formula are shown in Figure 3. First, we used the normal distribution sampling method to calculate the likelihood and used the Bayesian formula to calculate the posterior probability of every indicator. Second, we adopted the analytic hierarchy process (AHP) method to obtain the indicator weights and calculated the posterior probability of the multi-indicator comprehensive WRV assessment of each subsystem. Next, we used the AHP method again to calculate the weight of every subsystem, and we calculated the posterior probability of the multisubsystem WRV assessment of the integrated water resources system with the Bayesian formula. The calculation steps that were taken with the Bayesian method were as follows.

(1) Calculate the prior probability of every indicator that belongs to a certain standard type. Due to insufficient information about the indicators, this research assumed that the probability of each type was equal. Specifically, we had the following:

$$
P\left(y_{j 1}\right)=P\left(y_{j 2}\right)=P\left(y_{j 3}\right)=P\left(y_{j 4}\right)=P\left(y_{j 5}\right)=\frac{1}{5},
$$


where $P\left(y_{j i}\right)_{i=1,2, \ldots, 5}$ is the prior probability of the $j$ th indicator that belongs to the $i$ th standard type.

(2) Calculate the likelihood of every indicator. Here, we adopted the normal distribution sampling method to estimate the likelihoods. The calculation processes of the normal distribution sampling method were as follows.

(i) Calculate the variation coefficient $C_{v j}$ for the $j$ th indicator with (2):

$$
\begin{gathered}
a_{j}=\frac{\left(\sum_{i=1}^{5} y_{j i}\right)}{5}, \\
\sigma_{j}=\sqrt{\frac{\sum_{i=1}^{5}\left(y_{j i}-a_{j}\right)^{2}}{4}}, \\
C_{v j}=\frac{\sigma_{j}}{a_{j}},
\end{gathered}
$$

where $i$ is the number of standard types $(i=$ $1,2, \ldots, 5), j$ is the number of indicators of a subsystem $(j=1,2, \ldots, n), y_{j i}$ is the standard value of the $j$ th indicator of the $i$ th standard type, $a_{j}$ is the mean value of the normal distribution, and $\sigma_{j}$ is the standard deviation.

(ii) Use $C_{v j}$ to obtain the variation coefficient of the sampling values by assuming the following:

$$
C_{v j i}=C_{v j}
$$

where $C_{v j i}$ is the variation coefficient of the sampling values of the $i$ th type.

(iii) Calculate the standard deviation with (4), with taking $y_{j i}$ as the mean value of the $i$ th type

$$
\sigma_{j i}=C_{v j i} \cdot y_{j i}
$$

where $\sigma_{j i}$ is the standard deviation of the $i$ th type.

(iv) Standardize the sampling value with (5)

$$
t_{j k}=\frac{x_{j k}-y_{j i}}{\sigma_{j i}}
$$

where $x_{j k}$ is the value of the $j$ th indicator of the $k$ th subsystem, and $t_{j k}$ is the standardized value of $x_{j k}$.

(v) Calculate the likelihood $P\left(x_{j k} \mid y_{j i}\right)$ of every indicator with (6)

$$
P\left(x_{j k} \mid y_{j i}\right)=2\left(1-\Phi\left(\left|t_{j k}\right|\right)\right),
$$

where $k$ is the number of subsystems $(k=$ $1,2, \ldots, 4)$, and $\Phi\left(\left|t_{j k}\right|\right)=\int_{-\infty}^{t_{j k}}(1 / \sqrt{2 \pi}) e^{-u / 2} d u$.
(3) Calculate the posterior probability with (7):

$$
P\left(y_{j i} \mid x_{j k}\right)=\frac{P\left(y_{j i}\right) P\left(x_{j k} \mid y_{j i}\right)}{\sum_{i=1}^{5} P\left(y_{j i}\right) P\left(x_{j k} \mid y_{j i}\right)},
$$

where $P\left(y_{j i} \mid x_{j k}\right)$ is the likelihood of every indicator that belongs to $i$ th type.

(4) Calculate the comprehensive probability of the multiindicator WRV assessment of each subsystem with (8):

$$
P_{i}=\sum_{j=1}^{m} \omega_{j} P\left(y_{j i} \mid x_{j k}\right),
$$

where $P_{i}$ is the weighted sum of the posterior probability of the multi-indicator and represents the probability that WRV of the $k$ th subsystem belongs to the $i$ th standard type, $\omega_{j}$ is the weight of the $j$ th indicators, and $0 \leq \omega_{j} \leq 1, \sum_{j=1}^{m} \omega_{j}=1$. Here, we used the AHP method to determine the weight $\omega_{j}$ of every indicator of each subsystem.

(5) Calculate the posterior probability of the multisubsystem WRV assessment of the integrated water resources system using (8) again. Here, $P_{i}$ is the weighted sum of the posterior probability of four subsystems and represents the probability that WRV of the integrated water resources system belongs to the $i$ th standard type, $\omega_{j}$ is the weight of the $j$ th subsystem, and $0 \leq \omega_{j} \leq 1 ; \sum_{j=1}^{m} \omega_{j}=1, m=4$, $k=1$.

3.3. Other WRV Assessment Methods for Comparison. In order to verify the effectiveness of the proposed method and compare the results among different methods, we chose the other two WRV assessment methods for simultaneous calculation, as follows.

(1) The GRA Method. The GRA method is an impact evaluation model that measures the degree of similarity or difference between two sequences based on the level of relation [33]. The calculation steps are as follows.

(i) Compose a reference sequence (i.e., (9)) and an assessment sequence (i.e., (10)):

$$
\begin{aligned}
& x_{i}(k)=\left\{x_{i}(1), x_{i}(2), \ldots, x_{i}(n)\right\} \quad(i=1,2, \ldots, t), \\
& y_{j}(k)=\left\{y_{j}(1), y_{j}(2), \ldots, y_{j}(n)\right\} \quad(j=1,2, \ldots, m),
\end{aligned}
$$

where $n$ is the number of parameters, $m$ is the number of regions to be assessed, $t$ is the number of ranges of parameters, $x_{i}(k)$ is the observed value of the $k$ th parameter of the $i$ th range, and $y_{j}(k)$ is the representative value of the $k$ th parameter of the $j$ th region. 
(ii) Normalize the reference sequence with (11) and the assessment sequence with (12) and (13):

$$
x_{i}^{\prime}(k)=\left\{\begin{array}{l}
\frac{x_{i}(k)-x_{1}(k)}{x_{t}(k)-x_{1}(k)} \\
\frac{x_{1}(k)-x_{i}(k)}{x_{1}(k)-x_{t}(k)}
\end{array}\right.
$$

For an indicator for which the greater value means a higher vulnerability, normalization can be performed with (12):

$$
y_{j}^{\prime}(k)= \begin{cases}0, & y_{j}(k) \leq x_{1}(k) \\ \frac{y_{j}(k)-x_{1}(k)}{x_{t}(k)-x_{1}(k)}, & x_{1}(k)<y_{j}(k)<x_{t}(k) \\ 1, & y_{j}(k) \geq x_{t}(k) .\end{cases}
$$

For an indicator for which the smaller value means a higher vulnerability, normalization can be performed with (13):

$$
y_{j}^{\prime}(k)= \begin{cases}0, & y_{j}(k) \geq x_{1}(k) \\ \frac{x_{1}(k)-y_{j}(k)}{x_{1}(k)-x_{t}(k)}, & x_{1}(k)>y_{j}(k)>x_{t}(k) \\ 1, & y_{j}(k) \leq x_{t}(k) .\end{cases}
$$

(iii) Identify $\Delta_{j i}(k), \Delta_{\max }$, and $\Delta_{\text {min }}$ with (14):

$$
\begin{gathered}
\Delta_{j i}(k)=\left|y_{j}^{\prime}(k)-x_{i}^{\prime}(k)\right|, \\
\Delta_{\max }=\max \max \left|y_{j}^{\prime}(k)-x_{i}^{\prime}(k)\right|, \\
\Delta_{\text {min }}=\min \min \left|y_{j}^{\prime}(k)-x_{i}^{\prime}(k)\right|,
\end{gathered}
$$

where $\Delta_{j i}(k)$ is the difference between $y_{j}^{\prime}(k)$ and $x_{i}^{\prime}(k)$, and $\Delta_{\max }$ and $\Delta_{\min }$ represent the maximum and minimum values among all the $\Delta_{j i}(k)$, respectively.

(iv) Calculate the grey relational coefficient $\zeta_{j i}(k)$ :

$$
\zeta_{j i}(k)=\frac{\Delta_{\min }+0.5 \Delta_{\max }}{\Delta_{j i}(k)+0.5 \Delta_{\max }} .
$$

(v) Calculate the grey relational grade $r_{j i}$ :

$$
r_{j i}=\sum_{k=1}^{n} \lambda_{i} \zeta_{j i}(k),
$$

where $\lambda_{i}$ is the weight of the $i$ th parameter.

(vi) Determine the maximum value $r_{\max }$ of the grey relational grades. The range of $r_{\max }$ represents the vulnerability type which a region belongs to. For the multilayer indicator system, $r_{j i}$ for one layer should be regarded as $\zeta_{j i}$ for its next layer and then be recalculated with (16).
(2) The PS Method. The PS method can be used to calculate the vulnerability indicator, to identify the degree of the WRV. The steps that are taken with this method are as follows.

(i) Establish a reference system $C_{i}=\left(C_{1}, C_{2}, \ldots, C_{n}\right)$ that can be represented by the median of every indicator, and an assessment system $P_{i}=\left(P_{1}, P_{2}, \ldots, P_{n}\right)$ that uses the observed indicator values in the study region.

(ii) Normalize the values of the assessment system by (17) and (18).

For the indicator that the greater the value, the more vulnerable the system, normalization can be performed as follows:

$$
X_{i}=\frac{P_{i}}{C_{i}} .
$$

For the indicator that the smaller the value, the more vulnerable the system, normalization is performed as follows:

$$
X_{i}=\frac{C_{i}}{P_{i}}
$$

(iii) Calculate the WRV degree by (19):

$$
V_{i}=\lambda_{i} \times X_{i}
$$

where $V_{i}$ is the WRV value of a region and $\lambda_{i}$ is the weight of indicator.

For the multilayer indicator system, $V_{i}$ obtained from one layer appears as $X_{i}$ of its next layer; then we recalculate $V_{i}$ by using (19). Finally, we can obtain the WRV degree of the integrated water resource system. The greater the value of $V_{i}$ is, the more vulnerable the region is.

\section{Results and Discussion}

4.1. WRV Assessment Results of Subsystems and the Integrated Water Resources System Based on the Bayesian Method. To compare the calculation results of the Bayesian method with those of the PS method and the GRA method [19], we chose the corresponding data in 2003 (see Table 2). With (7), the probability that each indicator of the subsystems belonged to a certain WRV level were obtained. Then, the WRV probabilities of the subsystems were obtained with (8). For the indicators of the hydrological subsystem (Figure 4), the lower the indicator value was, the larger the tendency of a county to belong to a higher WRV was. Some WRV levels to which indicators of the subsystems belonged were the same for most indicators of all nine counties (i.e., High WRV for the mean annual precipitation, Extreme WRV for the groundwater recharge of unit area, and Medium WRV for the drought indicator), with the exception of two indicators (i.e., the annual relative variability of precipitation, and the mean water resources amount of unit area); see Figures $4\left(a_{1}\right), 4\left(a_{2}\right), 4\left(a_{3}\right), 4\left(a_{4}\right)$, and $4\left(a_{5}\right)$. However, the probabilities that each indicator belonged to some WRV 
TABLE 2: Actual values of indicators for study area.

\begin{tabular}{|c|c|c|c|c|c|c|c|c|c|c|}
\hline Subsystem & Indicator & 1 & 2 & 3 & 4 & 5 & 6 & 7 & 8 & 9 \\
\hline \multirow{5}{*}{ A } & $a_{1}$ & 387.20 & 406.40 & 406.40 & 409.10 & 405.70 & 373.60 & 456.50 & 385.80 & 446.80 \\
\hline & $a_{2}$ & 20.83 & 27.75 & 22.71 & 22.65 & 14.12 & 21.90 & 22.77 & 18.83 & 18.30 \\
\hline & $a_{3}$ & 4.52 & 6.84 & 5.60 & 6.56 & 6.50 & 4.06 & 7.90 & 8.27 & 3.45 \\
\hline & $a_{4}$ & 2.58 & 4.76 & 3.09 & 4.80 & 2.43 & 2.94 & 2.95 & 5.68 & 1.65 \\
\hline & $a_{5}$ & 3.00 & 2.42 & 2.35 & 2.79 & 2.05 & 2.67 & 2.21 & 2.19 & 1.82 \\
\hline \multirow{7}{*}{ B } & $\mathrm{b}_{1}$ & 68.54 & 474.45 & 294.77 & 458.45 & 438.82 & 271.44 & 667.83 & 438.90 & 652.50 \\
\hline & $b_{2}$ & 198.26 & 1158.04 & 1317.14 & 736.19 & 412.31 & 311.41 & 570.93 & 464.04 & 523.04 \\
\hline & $\mathrm{b}_{3}$ & 194.44 & 336.77 & 422.60 & 485.15 & 193.68 & 138.14 & 296.07 & 372.02 & 250.80 \\
\hline & $\mathrm{b}_{4}$ & 1.49 & 1.96 & 9.25 & 7.81 & 3.81 & 12.30 & 1.85 & 17.19 & 6.44 \\
\hline & $b_{5}$ & 0.87 & 0.98 & 0.94 & 0.93 & 0.96 & 1.09 & 1.37 & 0.96 & 0.99 \\
\hline & $\mathrm{b}_{6}$ & 174.41 & 67.61 & 106.64 & 100.58 & 48.33 & 45.46 & 48.19 & 86.01 & 33.69 \\
\hline & $b_{7}$ & 70.00 & 65.00 & 68.00 & 70.00 & 62.00 & 60.00 & 45.00 & 43.00 & 41.00 \\
\hline \multirow{6}{*}{ C } & $\mathrm{c}_{1}$ & 76.24 & 50.94 & 47.59 & 59.18 & 76.83 & 58.36 & 71.69 & 51.00 & 56.37 \\
\hline & $c_{2}$ & 180.86 & 191.40 & 129.31 & 90.61 & 280.19 & 82.88 & 362.39 & 505.88 & 48.66 \\
\hline & $c_{3}$ & 3.46 & 1.13 & 0.32 & 2.56 & 0.40 & 0.42 & 0.23 & 1.47 & 0.62 \\
\hline & $\mathrm{c}_{4}$ & 49.82 & 50.03 & 71.39 & 63.65 & 61.54 & 89.99 & 42.86 & 0 & 66.64 \\
\hline & $c_{5}$ & 65.74 & 63.32 & 60.37 & 70.78 & 63.57 & 63.82 & 64.51 & 62.18 & 50.38 \\
\hline & $\mathrm{c}_{6}$ & 70.00 & 50.00 & 65.00 & 68.00 & 50.00 & 55.00 & 35.00 & 35.00 & 30.00 \\
\hline \multirow{3}{*}{$\mathrm{D}$} & $\mathrm{d}_{1}$ & 61.74 & 60.77 & 32.52 & 58.32 & 23.83 & 32.98 & 47.16 & 58.28 & 36.29 \\
\hline & $\mathrm{d}_{2}$ & 7.10 & 52.48 & 13.79 & 56.81 & 39.41 & 19.77 & 1.07 & 159.78 & 1.06 \\
\hline & $\mathrm{d}_{3}$ & 89.64 & 82.62 & 76.07 & 81.47 & 56.2 & 78.58 & 88.00 & 60.20 & 75.00 \\
\hline
\end{tabular}

Note: The symbolic descriptions for the 1st column of $A \sim D$ and the 2 nd column of $a_{1} \sim d_{3}$ are the same as those in Table 1; administrative divisions 1-9 are shown in Figure 1.

level were different for the nine counties, for example, a probability of $40.08 \%-46.51 \%$ belonging to High WRV for the mean annual precipitation, while $26.56 \%-36.49 \%$ belonging to Extreme WRV for the groundwater recharge of unit area. For the indicator of the mean water resources amount of unit area, six counties belonged to Extreme WRV, with the exception of Huai'an, Zhuolu, and Huailai Counties, which belonged to High WRV (see Figure $4\left(a_{3}\right)$ ). The WRV assessment results of the hydrological subsystem are shown in Figure 4(A). We can see that all of the nine counties had a high probability of belonging to High WRV (ranging from $27.07 \%$ to $33.48 \%$ ), followed by Medium WRV (22.3\%-25.4\%) and Extreme WRV (14.4\%-24.5\%). This finding meant that the WRV of the hydrological subsystem of the Zhangjiakou region was high. The hydrological subsystem reflected the natural situations of regional water resources. The abundance, stability, and hydrographic and meteorological conditions had a comprehensive impact on the WRV degree of the hydrological subsystem. Being located in arid and semiarid areas, water supply available of the Zhangjiakou region was insufficient, although the stability of water resources system was not too bad. Specifically, water abundance and groundwater recharge capacity were very weak, resulting in a high WRV probability of the hydrological subsystem. The results were consistent with those of the GRA method and the actual situations [19].

For indicators of the socioeconomic subsystem (Figure 5), water resources per capita of eight counties were low (approximately $399 \mathrm{~m}^{3}$ ), which had a high probability of belonging to Extreme WRV (26.67\%-54.02\%), except for Zhuolu County, which tended to belong to High WRV $(30.80 \%)$ (see Figure $5\left(b_{1}\right)$ ). This finding meant that the regional population increase had put great pressure on the water resources system. For the other indicators except for the water resources per capita, there were different WRV levels for each county. In general, the repetitive water utilization ratio of industry in this region tended to be Low WRV or Medium WRV (89\% altogether), which meant that the industrial water resources consumption was not serious. It is partly because the economic development mode of the region was mainly based on agriculture. Although the water supply and demand ratio for counties had a tendency to belong to Low WRV (11.95\%-41.20\%) or No WRV $(43.06 \%-$ $87.89 \%)$ from Figure $5\left(b_{5}\right)$, the water demand had been met by overextracting groundwater for a long time by the analysis combined with Figure $4\left(\mathrm{a}_{4}\right)$. In this way, the water resources use is not sustainable. Through the probability calculation with (7), while Yuxian and Chongli counties tended to belong to Low WRV, Yangyuan and Chongli counties belonged to No WRV while the other counties belonged to Extreme WRV (see Figure 5(B)). It can be concluded that the rapid increase of population and low water utilization efficiency resulted in a high WRV of socioeconomic subsystem. As a basic water resources security concern, the groundwater overdraft needs more attention for the purpose of sustainable water use in the future, although it temporarily alleviated the shortage of water resources.

For the indicators of the ecoenvironmental subsystem (Figure 6), the most possible two WRV levels for the indicator of the ratio of soil and water loss were the same for all nine 


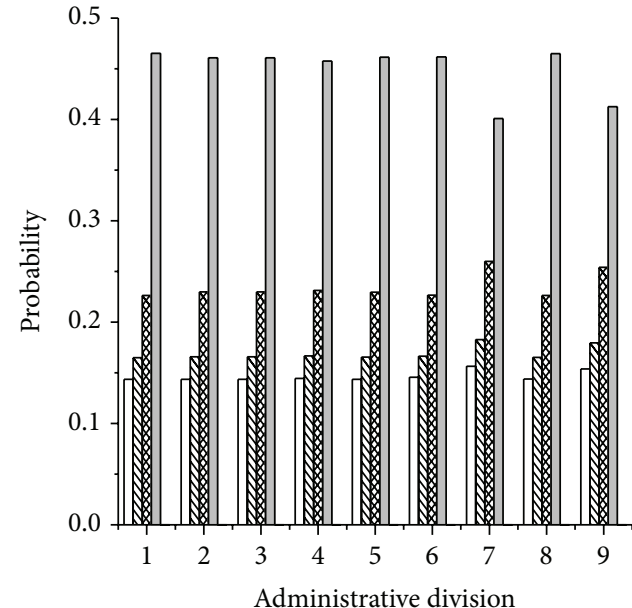

$\left(\mathrm{a}_{1}\right)$ Mean annual precipitation

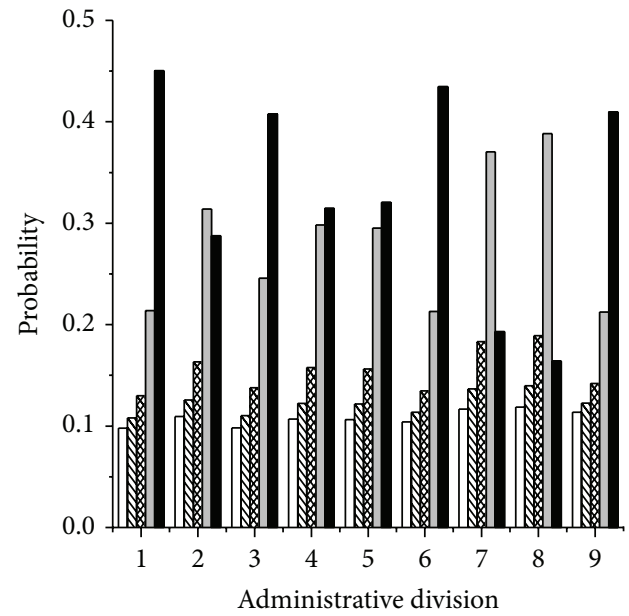

$\left(\mathrm{a}_{3}\right)$ Mean water resources
amount of unit area
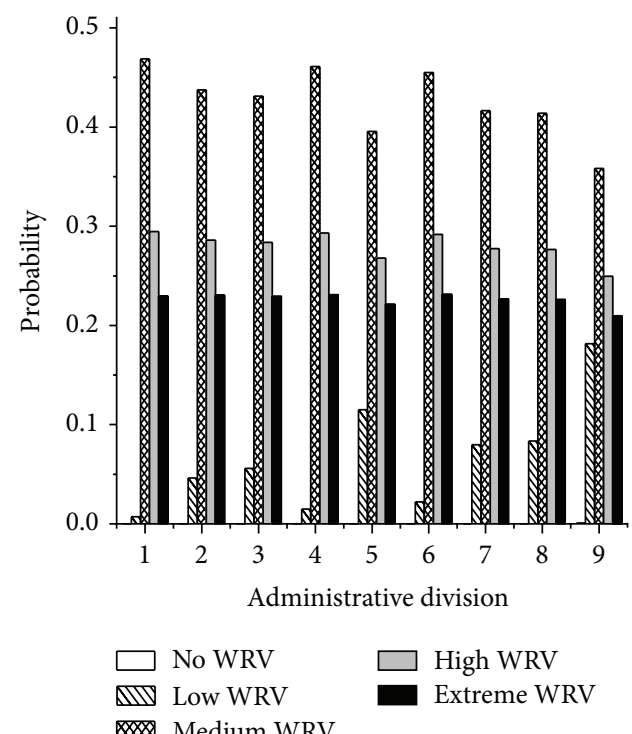

$\left(a_{5}\right)$ Drought index

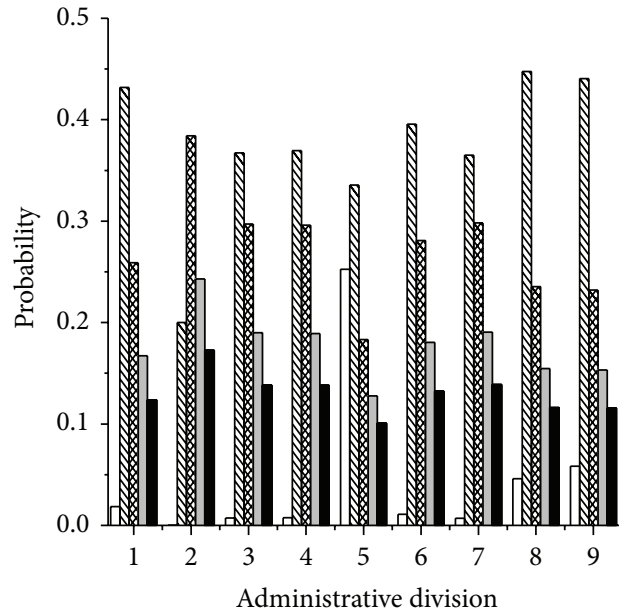

$\left(\mathrm{a}_{2}\right)$ Annual relative variability of precipitation

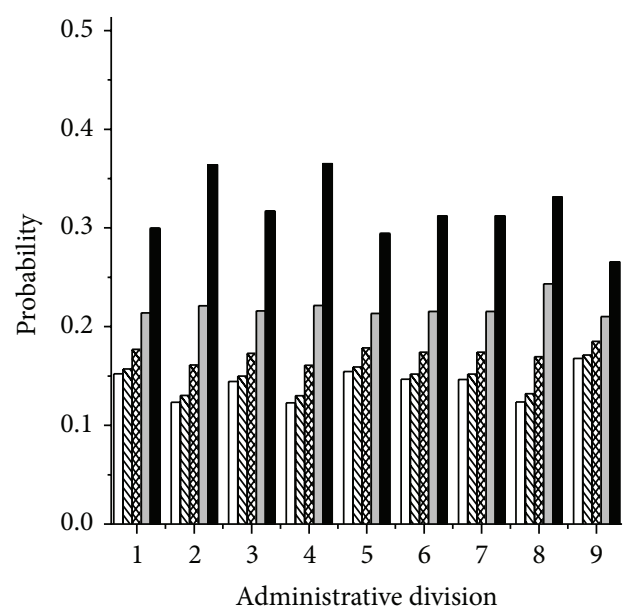

$\left(a_{4}\right)$ Groundwater recharge of unit area
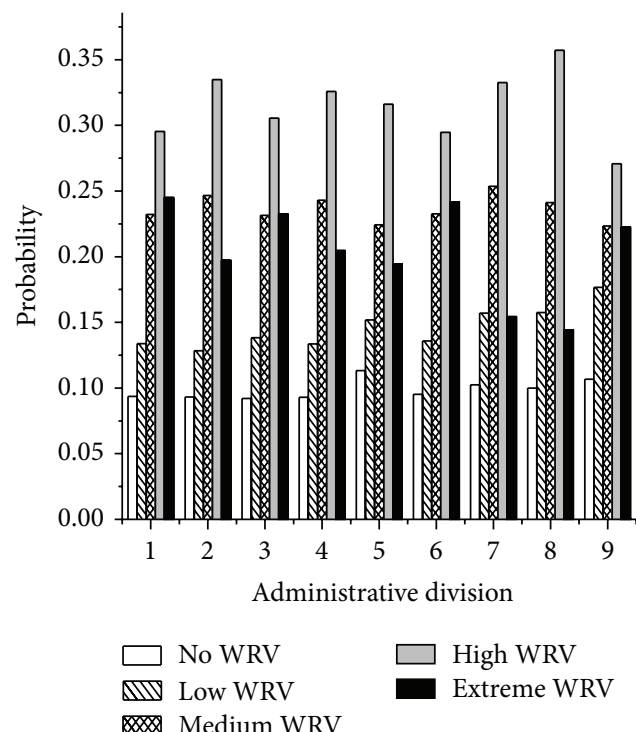

(A) Hydrological subsystem

FIGURE 4: Each indicator's WRV and integrated WRV of the hydrological subsystem in the Zhangjiakou region (administrative divisions 1-9 are shown in Figure 1). 


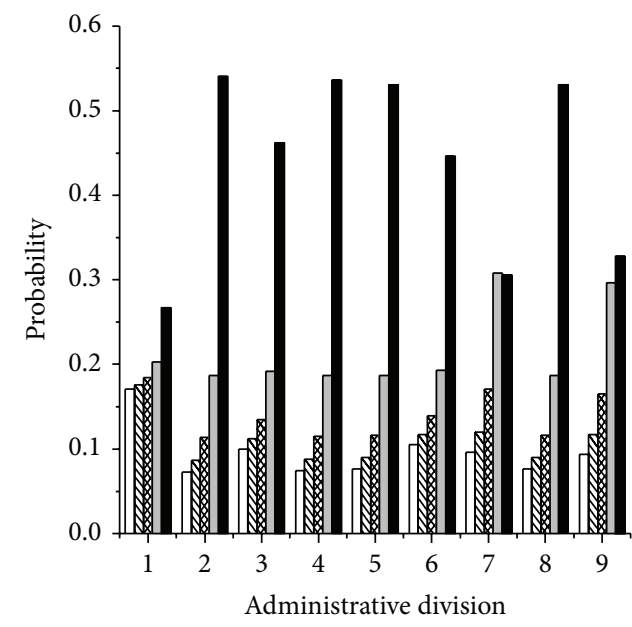

$\left(b_{1}\right)$ Water resources per capita

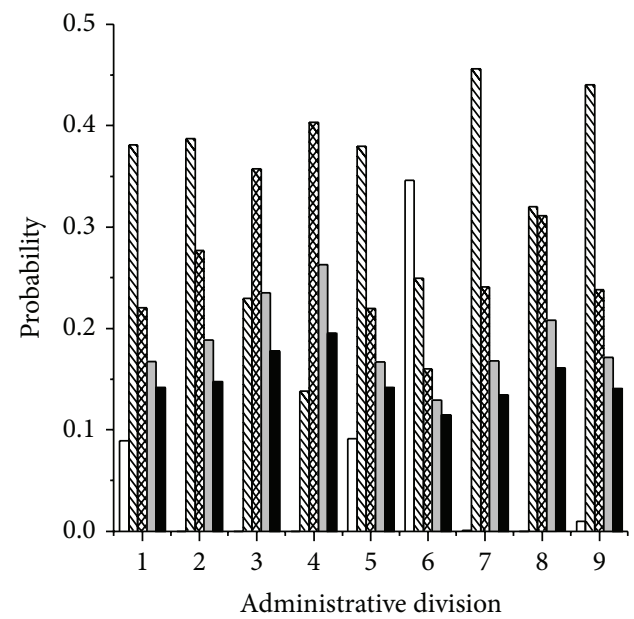

$\left(b_{3}\right)$ Water consumption per capita
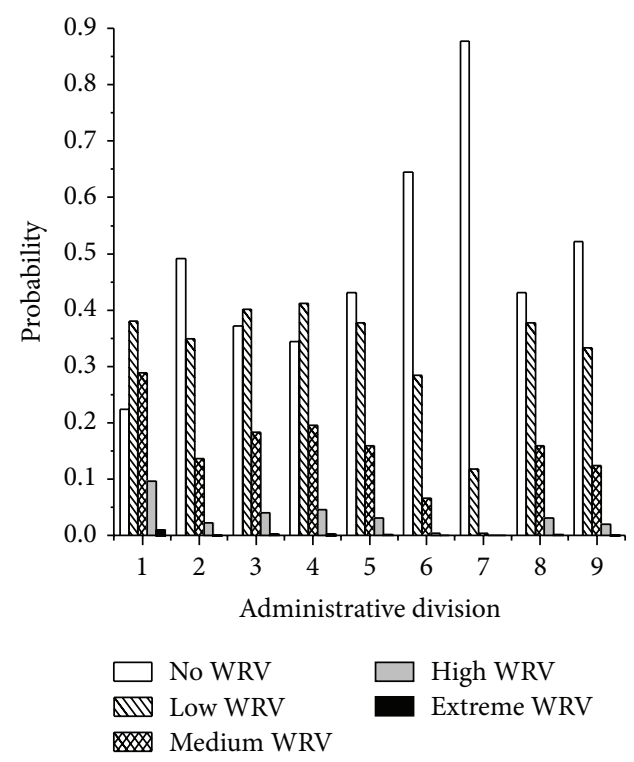

$\left(b_{5}\right)$ Water supply and demand ratio

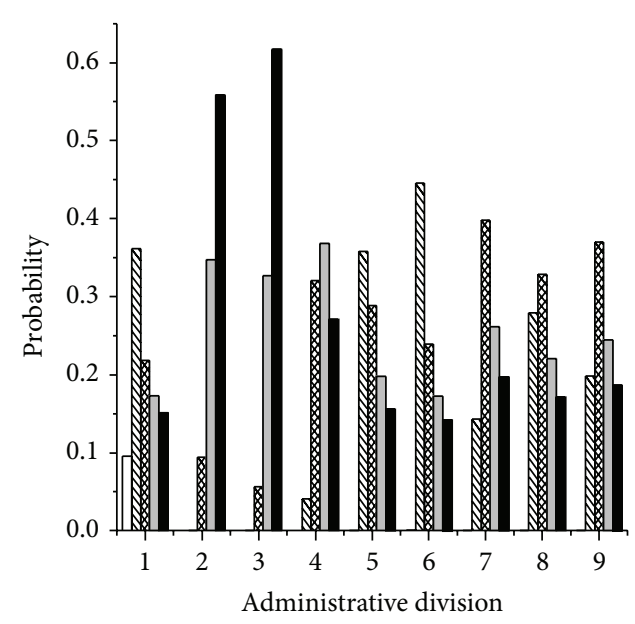

$\left(b_{2}\right)$ Water consumption per unit GDP

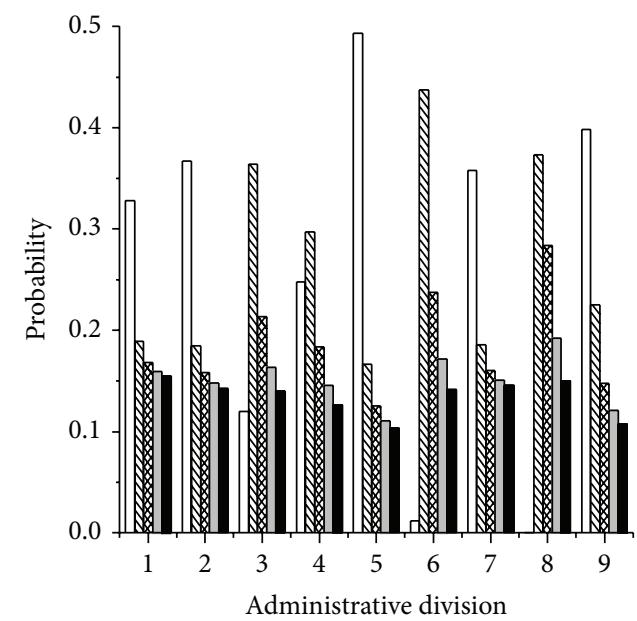

$\left(b_{4}\right)$ Difference between planned and actual water utilization amount
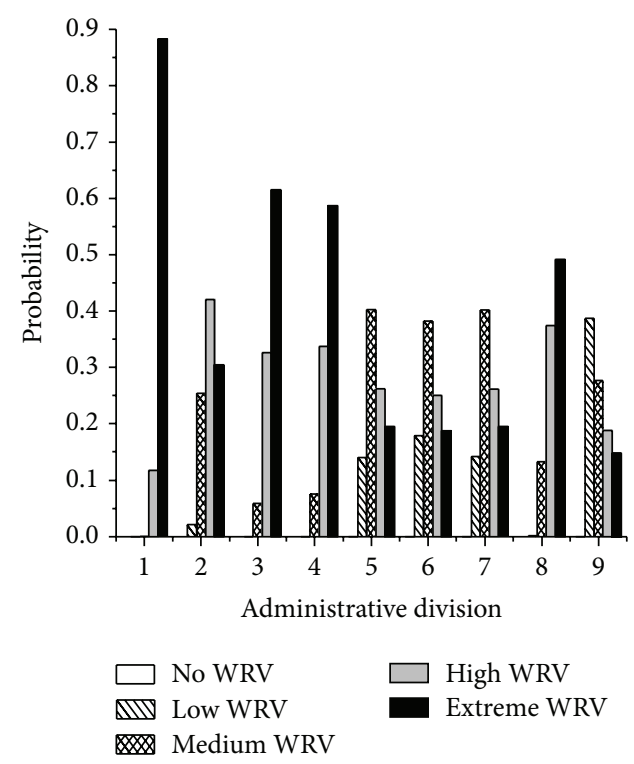

$\left(b_{6}\right)$ Water resources development and utilization ratio

Figure 5: Continued. 

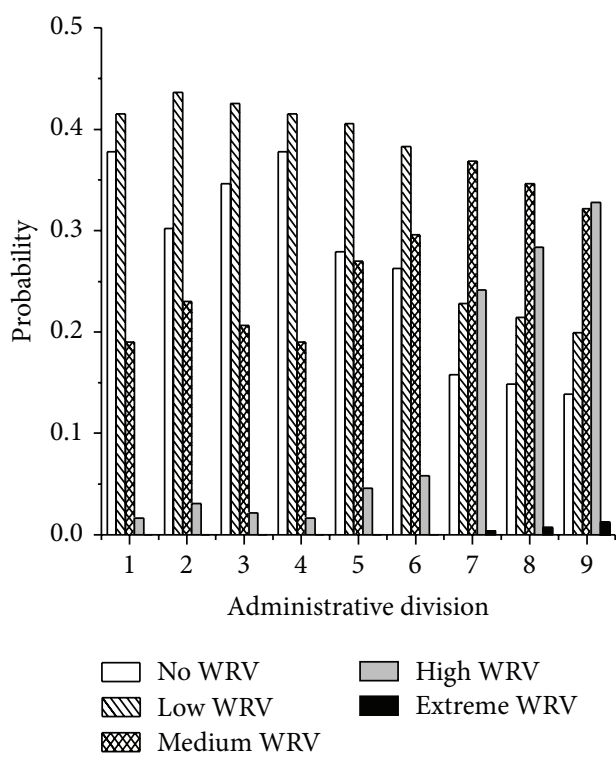

$\left(b_{7}\right)$ Repetitive utilization ratio of industrial water
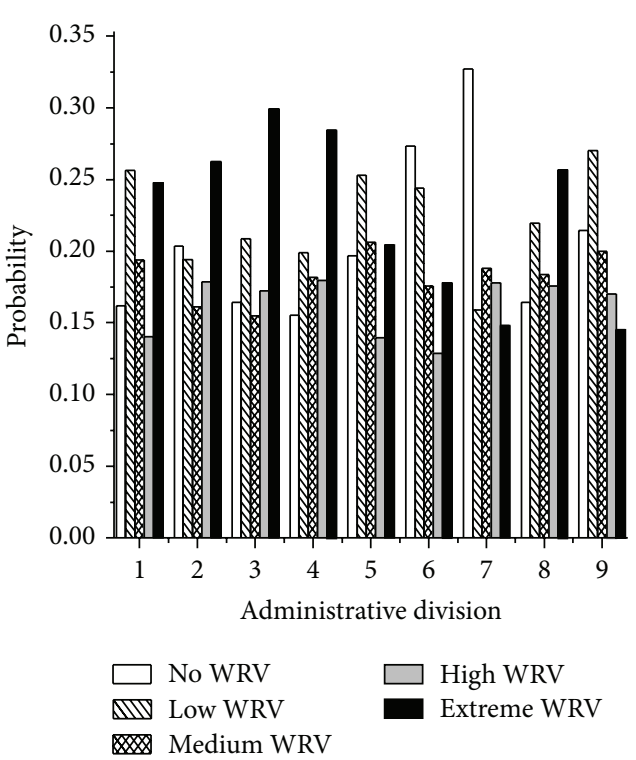

(B) Socioeconomic subsystem

FIGURE 5: Each indicator's WRV and integrated WRV of the socioeconomic subsystem in the Zhangjiakou region (administrative divisions 1-9 are shown in Figure 1).

counties (i.e., Extreme WRV ranked first with a high probability of $62.54 \%-79.18 \%$, and High WRV ranked second). The WRV levels were different from each other for other indicators, including the land development ratio, the fertilizer use intensity, the pollution index of surface water, the ratio of polluted groundwater exceeding the standard, and the disposal ratio of sewage, due to their respective ecoenvironment conditions. Because most of the large counties were located in remote mountainous areas, their fertilizer use intensity was not excessively large. Therefore, as for the indicator of the fertilizer use intensity, except for the fact that Huailai County tended to belong to High WRV, others belonged to Medium WRV (Figure 6( $\left.c_{2}\right)$ ). In addition, the pressure of economic development had greatly aggravated the pollution of the surface water, leading to a high probability for the indicator of the pollution of the surface water belonging to Medium WRV (39.52\%), High WRV (38.68\%-51.08\%), and Extreme WRV (77.68\%-99.99\%) (Figure 6(c $\left.c_{3}\right)$ ). For example, all of the three most prosperous counties in this region, that is, Zhangjiakou City, Xuanhua County, and Huailai County, had Extreme WRV. Among the nine counties, Zhangjiakou City had the largest population and the largest economic output. Except for the fact that the indicator of the disposal ratio of sewage for Zhangjiakou City had a Low WRV level, its WRV levels for the other indicators were usually higher by more than 1-2 levels compared with other counties, leading to an Extreme WRV for the comprehensive assessment of the ecoenvironmental subsystem. Overall, the ecological environment of the Zhangjiakou region was very vulnerable; except for Zhuolu County belonging to the Medium WRV, all of the other counties belonged to the Extreme WRV or High WRV (Figure 6(C)). With the rapid development of industry and agriculture, waste water discharge and extensive use of fertilizers and pesticides in agricultural production had increased, leading to the deterioration of water environment in Zhangjiakou region. In addition, polluted groundwater would cause the deterioration in ecoenvironment. All these factors had resulted in WRV of ecoenvironmental subsystem. To decrease the WRV, an extensive mode of economic development should be changed.

For the indicators of the hydraulic engineering subsystem (Figure 7), the water-saving irrigation ratio was usually high, with $67 \%$ of the counties belonging to Medium WRV or Low WRV and 22\% belonging to High WRV (Wanquan County and Yangyuan County) (see Figure $7\left(\mathrm{~d}_{1}\right)$ ). Because the situations of water resources and hydraulic engineering were different for the nine counties, the WRV for the indicators of the regulating capacity of the surface water for hydraulic engineering and storage was different (see Figure $7\left(\mathrm{~d}_{2}\right)$ ). For the indicator of the storage and release capacity of reservoirs, except for Huailai County belonging to Low WRV and Yuxian County belonging to Medium WRV, the other counties belonged to No WRV (see Figure $7\left(\mathrm{~d}_{3}\right)$ ). Overall, hydraulic engineering played an important role in the decline of the WRV; more than $50 \%$ of the counties, including Zhangjiakou City, Huai'an County, Xuanhua County, Zhuolu County, and Huailai County, had Low or No WRV in this region due to the reallocation and adjustment of the flood by means of water conservancy facilities (see Figure $7(\mathrm{D})$ ).

With (8), the WRV of the integrated water resources system of the Zhangjiakou region was obtained (see Figure 8). We can see the following: (1) WRV of the ecoenvironmental subsystem of Zhangjiakou City and XuanHua County was more vulnerable than other counties, which led to a high tendency to belong to Extreme WRV (26.8\% and 25.0\%, resp.); other counties belonged to High WRV ranging from $24.6 \%$ to $27.0 \%$; (2) there were no areas belonging to No WRV, Low WRV, and Medium WRV, indicating that the WRV of the 


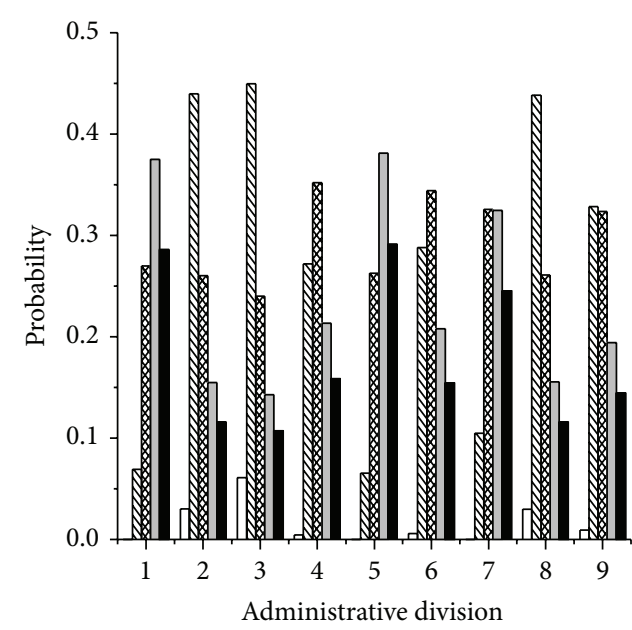

$\left(c_{1}\right)$ Land development ratio

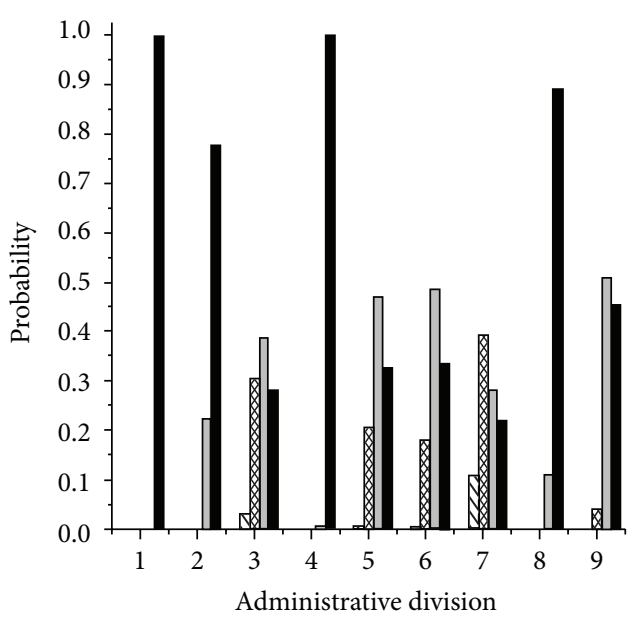

$\left(c_{3}\right)$ Pollution index of surface water
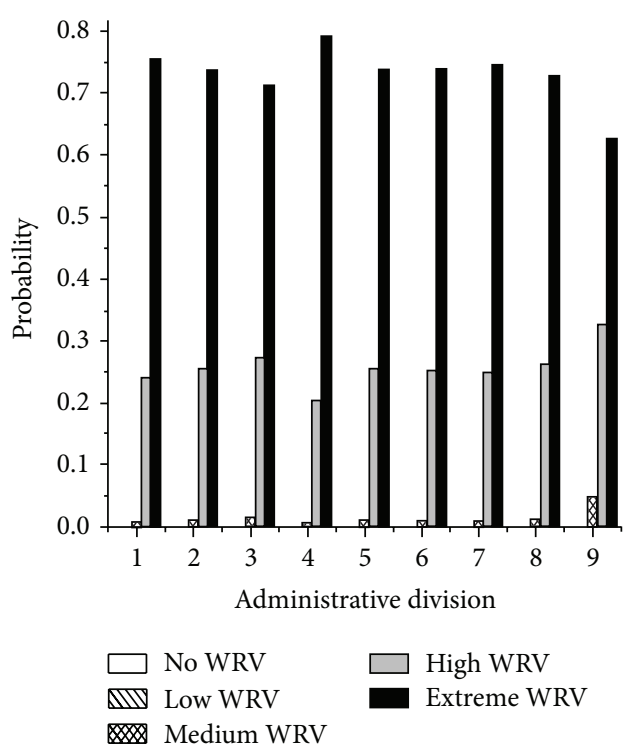

$\left(c_{5}\right)$ Ratio of soil and water loss

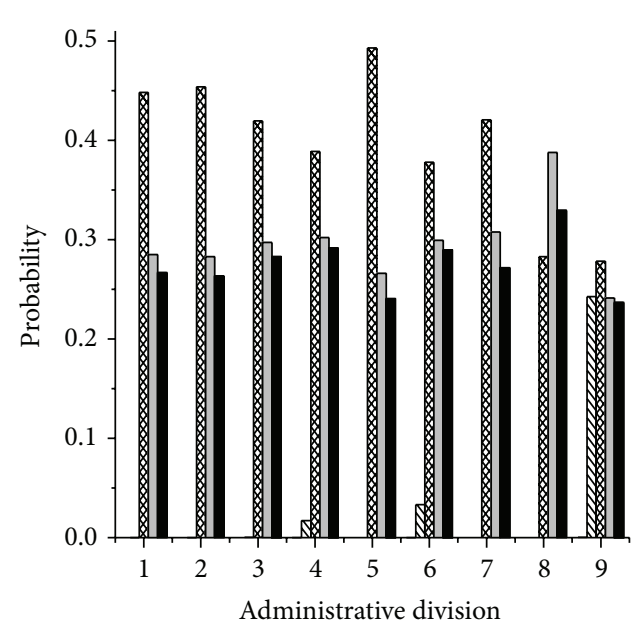

$\left(c_{2}\right)$ Fertilizer use intensity

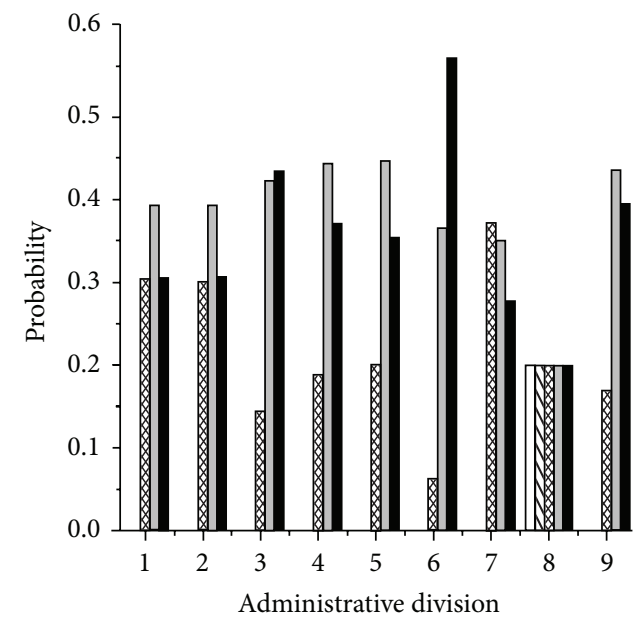

$\left(c_{4}\right)$ Ratio of polluted groundwater exceeding the standard
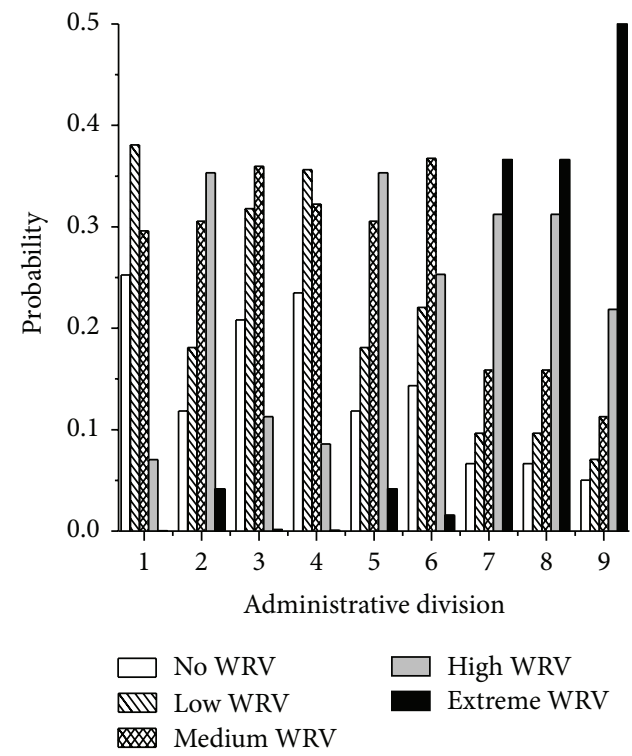

$\left(c_{6}\right)$ Disposal ratio of sewage

Figure 6: Continued. 


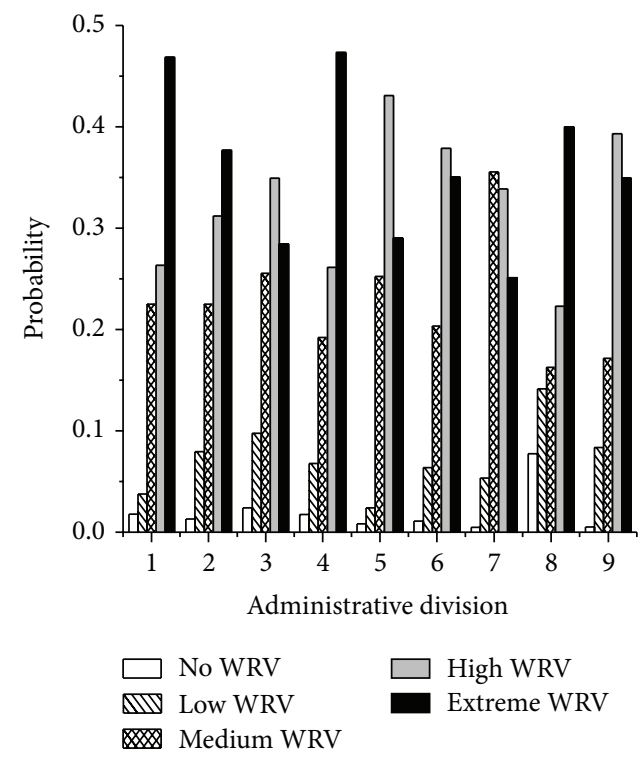

(C) Ecoenvironmental subsystem

FIGURE 6: Each indicator's WRV and integrated WRV of the ecoenvironmental subsystem in the Zhangjiakou region (administrative divisions 1-9 are shown in Figure 1).

TABLE 3: Results comparison between the Bayesian method, the GRA method, and the PS method for the integrated water resources system.

\begin{tabular}{|c|c|c|c|}
\hline Administrative division & Bayesian method & GRA method & PS method \\
\hline Zhangjiakou City & Extreme WRV & Extreme WRV & Extreme WRV \\
\hline Huaian County & High WRV & High WRV & Extreme WRV \\
\hline Wanquan County & High WRV & High WRV & Extreme WRV \\
\hline Xuanhua County & Extreme WRV & High WRV & Extreme WRV \\
\hline Yuxian County & High WRV & High WRV & Extreme WRV \\
\hline Yangyuan County & High WRV & High WRV & Extreme WRV \\
\hline Zhuolu County & High WRV & High WRV & High WRV \\
\hline Huailai County & High WRV & High WRV & Extreme WRV \\
\hline Chongli County & High WRV & High WRV & Extreme WRV \\
\hline
\end{tabular}

integrated water resources system of the Zhangjiakou region was very serious. WRV was an objective attribute of the water resources system, whose vulnerability came from its internal characteristics and external driving forces. The assessment results above were consistent with the actual situation of the water resources in the Zhangjiakou region, which showed that the Bayesian method can be well used for regional WRV assessment.

4.2. Results Comparison with the PS Method and the GRA Method. A comparison of the assessment results among the Bayesian method, the GRA method, and the PS method was conducted (Table 3). It can be seen that the WRV of 6 regions with the PS method was higher than those with the Bayesian method, and 7 regions were higher than those with the GRA method. The PS method had the advantages of a simple principle and simple linear calculation procedure for both the assessment and reference systems, but it can easily generate distorted results due to magnifying the influence of some indicators, and the resulting WRV levels were higher than those use the other two methods.

The results of the Bayesian method and the GRA method were almost the same, with the exception of the assessment for Xuanhua County. The two methods divided the sample data into several grades, to determine the vulnerable degree of the water resources in a large range, which can fully reflect the uncertainties in the water resources system. However, the GRA method had a high requirement of assessment and standard data. The assessment results for WRV were the possibility or tendency of the adverse impact of natural disasters and human activities on the water resources system, which can reflect the uncertain and fuzzy characteristics of the WRV reasonably. At the same time, it was very simple and quick, which made it more convenient and reliable for the WRV assessment. In the practical calculation, multiple methods should be used comprehensively for WRV assessment, to provide a more reliable decision-making basis for water resources management. 


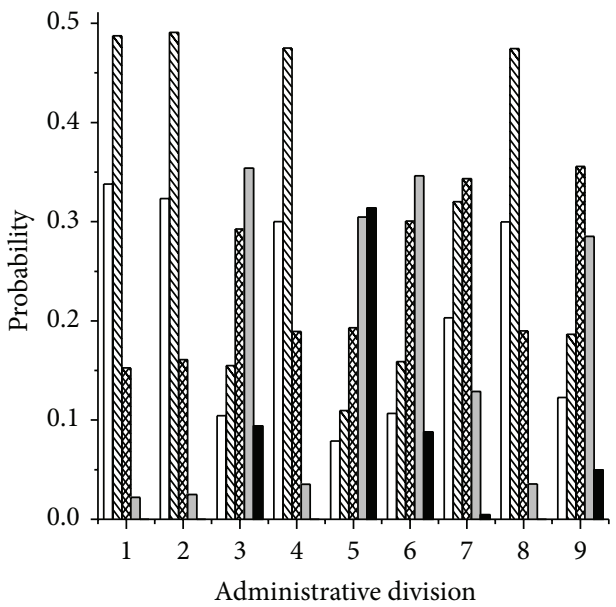

$\left(\mathrm{d}_{1}\right)$ Water-saving irrigation ratio
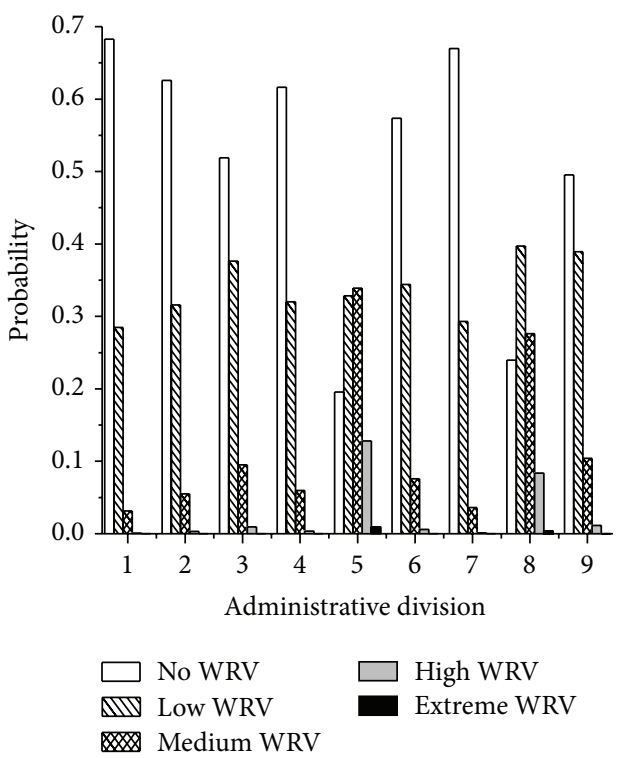

$\left(d_{3}\right)$ Ratio of effective storage to total storage of reservoirs

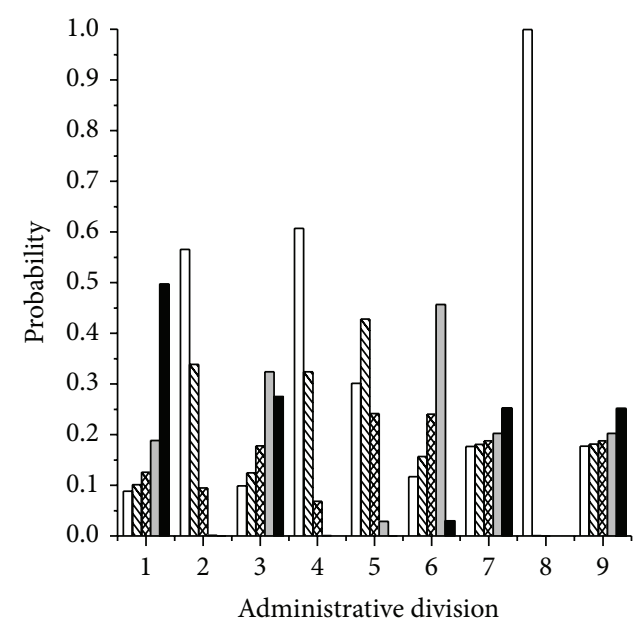

$\left(d_{2}\right)$ Ratio of design utilizable storage of reservoir to surface water quantity
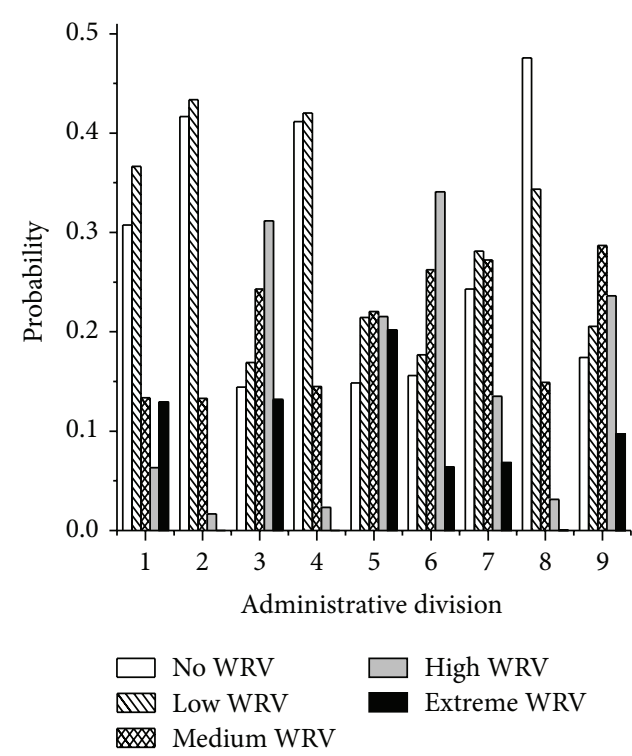

(D) Hydraulic engineering subsystem

FIGURE 7: Each indicator's WRV and integrated WRV of the hydraulic engineering subsystem in the Zhangjiakou region (administrative divisions 1-9 are shown in Figure 1).

\section{Conclusions}

A Bayesian method that used the sampling normal distribution method and the AHP method was developed to assess the WRV of the Zhangjiakou region. The results showed that (1) the vulnerability of the region was very high; Zhangjiakou City and Xuanhua County belonged to Extreme WRV with the probabilities of $26.8 \%$ and $25.0 \%$, respectively, while other counties belonged to High WRV (24.6\%-27.0\%). This finding indicated that the socioeconomic development had put a large amount of pressure on the water resources. Under the approximately same natural conditions for these nine adjacent areas, the economy developed more rapidly, and the WRV will become more serious. (2) Additionally, the results of WRV assessment of the Zhangjiakou region by using the
Bayesian method were consistent with the actual situation. This finding indicated that the Bayesian method was feasible for the WRV assessment with a simple calculation process.

The Zhangjiakou region undertook the important mission of providing a water supply for Beijing City, but the water resources condition was not good. Water scarcity and water pollution had seriously affected the sustainable development of the regional agricultural production and economic development. To reduce the WRV and enhance the ability of self-recovery of the water environment and to improve sustainable utilization of water resources of the Zhangjiakou region, effective countermeasures must be taken from the following aspects: (1) developing a water-saving industry and technology for the sewage purification, to improve the reuse level of the waste water and to construct a water-saving 


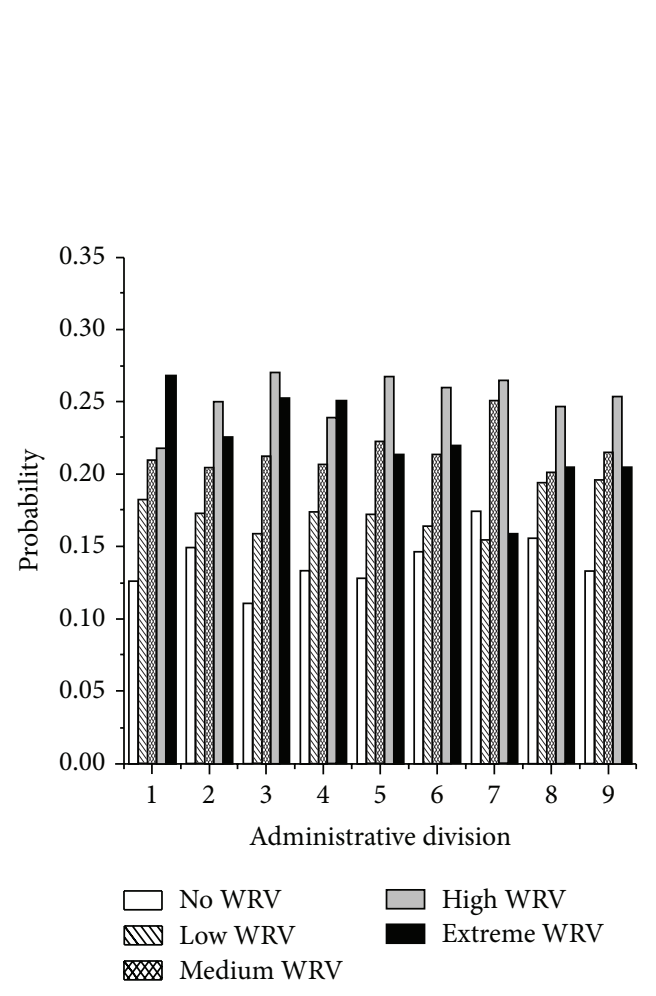

(a)

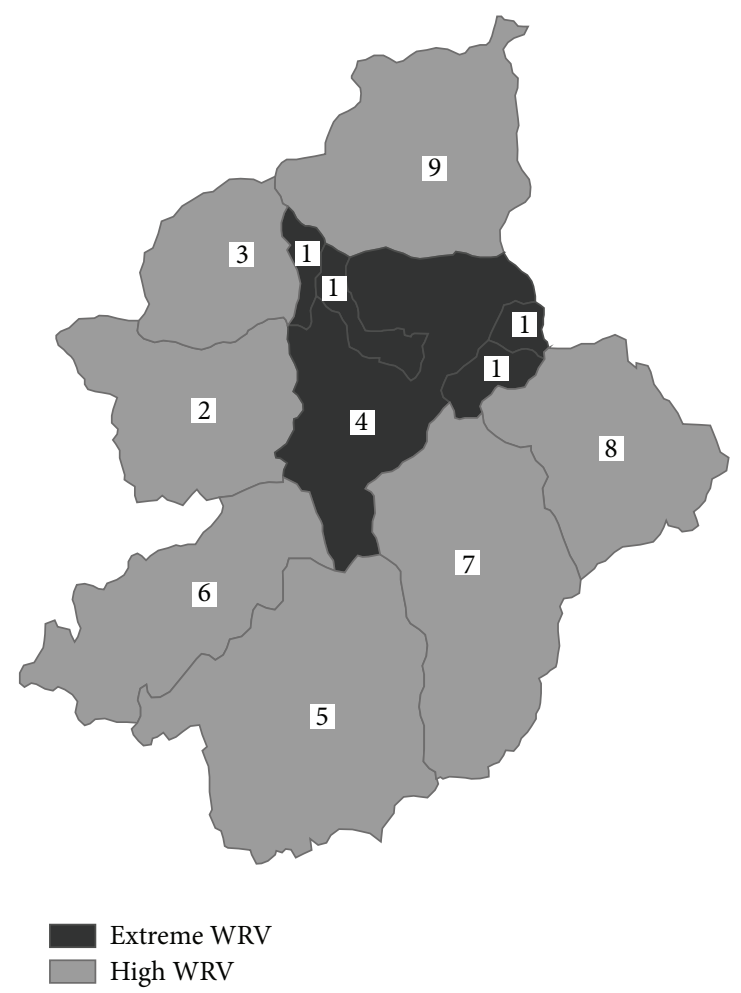

(b)

FIGURE 8: WRV of the integrated water resources system in the Zhangjiakou region: (a) WRV probabilities; (b) WRV spatial distribution (administrative divisions 1-9 are shown in Figure 1).

society, which can reduce the water environment deterioration and shortage of water resources in the Zhangjiakou region; (2) increasing the vegetation coverage, which would enhance the capacity for soil and water preservation, and strengthening controls on nonpoint pollution and population growth, to promote the sustainable development of a social economy and ecoenvironment; (3) raising public environmental awareness for the effective protection and sustainable utilization of water resources.

Due to insufficient information, we assumed that the prior probability of an indicator that belonged to the different vulnerability grades was equal. However, their prior probabilities were different in practice. The quantity of indicator data that was available largely limits the accuracy of the assessment. Thus, acquiring more indicator data could help to improve the precision of the WRV assessment. In addition, there is a threshold of the damage to the water resources system; the WRV assessment can provide only a level range (such as No WRV and Extreme WRV), and it is a future task to determine the damage threshold through a mechanism study.

\section{Conflict of Interests}

The authors declare that there is no conflict of interests regarding the publication of this paper.

\section{Acknowledgments}

The research was funded by the National Science Foundation for Innovative Research Group (no. 51121003), and the National Natural Science Foundation of China (50509001). The authors would like to extend special thanks to the editor and the anonymous reviewers for their valuable comments in greatly improving the quality of this paper.

\section{References}

[1] Z. J. Li, Q. W. Chen, Q. Xu, and K. Blanckaert, "Generalized likelihood uncertainty estimation method in uncertainty analysis of numerical eutrophication models: take bloom as an example," Mathematical Problems in Engineering, vol. 2013, Article ID 701923, 9 pages, 2013.

[2] X. Wang, F. B. Ma, and J. Y. Li, "Water resources vulnerability assessment based on the parametric-system method: a case study of the Zhangjiakou region of guanting reservoir Basin, North China," Procedia Environmental Sciences, vol. 13, pp. 1204-1212, 2012.

[3] K. Jinno, Z. X. Xu, A. Kawamura, and K. Tajiri, "Risk assessment of water supply system during drought," International Journal of Water Resources Development, vol. 11, no. 2, pp. 185-204, 1995.

[4] Z. Xu, K. Jinno, A. Kawamura, S. Takesaki, and K. Ito, "Performance risk analysis for Fukuoka water supply system," Water Resources Management, vol. 12, no. 1, pp. 13-30, 1998. 
[5] Z. X. Xu, K. Takeuchi, H. Ishidaira, and X. W. Zhang, "Sustainability analysis for Yellow River water resources using the system dynamics approach," Water Resources Management, vol. 16, no. 3, pp. 239-261, 2002.

[6] L. L. Liu, "Concept and quantitative assessment of vulnerability of water resource," Bulletin of Soil and Water Conservation, vol. 22, no. 2, pp. 41-44, 2002 (Chinese).

[7] J. D. Arthur, H. A. R. Wood, A. E. Baker, J. R. Cichon, and G. L. Raines, "Development and implementation of a Bayesian-based aquifer vulnerability assessment in Florida," Natural Resources Research, vol. 16, no. 2, pp. 93-107, 2007.

[8] B. Kattaa, W. Al-Fares, and A. R. Al Charideh, "Groundwater vulnerability assessment for the Banyas Catchment of the Syrian coastal area using GIS and the RISKE method," Journal of Environmental Management, vol. 91, no. 5, pp. 1103-1110, 2010.

[9] A. Sorichetta, M. Masetti, C. Ballabio, S. Sterlacchini, and G. P. Beretta, "Reliability of groundwater vulnerability maps obtained through statistical methods," Journal of Environmental Management, vol. 92, no. 4, pp. 1215-1224, 2011.

[10] A. Sorichetta, M. Masetti, C. Ballabio, and S. Sterlacchini, "Aquifer nitrate vulnerability assessment using positive and negative weights of evidence methods, Milan, Italy," Computers and Geosciences, vol. 48, pp. 199-210, 2012.

[11] D. Mirauda and M. Ostoich, "Surface water vulnerability assessment applying the integrity model as a decision support system for quality improvement," Environmental Impact Assessment Review, vol. 31, no. 3, pp. 161-171, 2011.

[12] C. Yu, Y. Yao, G. Hayes, B. Zhang, and C. Zheng, "Quantitative assessment of groundwater vulnerability using index system and transport simulation, Huangshuihe catchment, China," Science of the Total Environment, vol. 408, no. 24, pp. 6108-6116, 2010.

[13] J. J. Martínez-Bastida, M. Arauzo, and M. Valladolid, "Intrinsic and specific vulnerability of groundwater in central Spain: the risk of nitrate pollution," Hydrogeology Journal, vol. 18, no. 3, pp. 681-698, 2010.

[14] J. Zhou, G. Li, F. Liu, Y. Wang, and X. Guo, "DRAV model and its application in assessing groundwater vulnerability in arid area: a case study of pore phreatic water in Tarim Basin, Xinjiang, Northwest China," Environmental Earth Sciences, vol. 60, no. 5, pp. 1055-1063, 2010.

[15] P. Martínez-Santos, M. R. Llamas, and P. E. Martínez-Alfaro, "Vulnerability assessment of groundwater resources: a modelling-based approach to the Mancha Occidental aquifer, Spain," Environmental Modelling and Software, vol. 23, no. 9, pp. 11451162, 2008.

[16] C. Butscher and P. Huggenberger, "Enhanced vulnerability assessment in karst areas by combining mapping with modeling approaches," Science of the Total Environment, vol. 407, no. 3, pp. 1153-1163, 2009.

[17] A. K. Antonakos and N. J. Lambrakis, "Development and testing of three hybrid methods for the assessment of aquifer vulnerability to nitrates, based on the drastic model, an example from NE Korinthia, Greece," Journal of Hydrology, vol. 333, pp. 288-304, 2007.

[18] H.-N. Zhu, X.-Z. Yuan, J. Liang, G.-M. Zeng, and H.-W. Jiang, "An integrated model for assessing the risk of water environmental pollution based on fuzziness," China Environmental Science, vol. 31, no. 3, pp. 516-521, 2011 (Chinese).

[19] J. Y. Li, Study on vulnerability assessment of water resources of guanting reservoir basin [M.S. thesis], Beijing Normal University, Beijing, China, 2007, (chinese).
[20] F. B. Ma, X. Wang, and C. H. Li, "Research progress in water resources vulnerability assessment," Journal of Water Resources and Water Engineering, vol. 23, no. 1, pp. 30-37, 2012 (Chinese).

[21] F. Zong, H. G. Xu, and H. Y. Zhang, "Prediction for traffic accident severity: comparing the Bayesian network and regression models," Mathematical Problems in Engineering, vol. 2013, Article ID 475194, 9 pages, 2013.

[22] T. Chan, H. Ross, S. Hoverman, and B. Powell, "Participatory development of a Bayesian network model for catchment-based water resource management," Water Resources Research, vol. 46, no. 7, Article ID W07544, 2010.

[23] S. S. Qian, A. Schulman, J. Koplos, A. Kotros, and P. Kellar, "A hierarchical modeling approach for estimating national distributions of c hemicals in public drinking water systems," Environmental Science and Technology, vol. 38, no. 4, pp. 1176$1182,2004$.

[24] R. Wu, S. S. Qian, F. Hao, H. Cheng, D. Zhu, and J. Zhang, "Modeling contaminant concentration distributions in China's centralized source waters," Environmental Science \& Technology, vol. 45, no. 14, pp. 6041-6048, 2011.

[25] F. B. Ma, C. H. Li, X. Wang, Z. F. Yang, C. C. Sun, and P. Y. Liang, "A Bayesian method for comprehensive water quality evaluation of the Danjiangkou Reservoir water source area, for the middle route of the South-to-North Water Diversion Project in China," Frontiers of Earth Science, vol. 8, no. 2, pp. 242-250, 2014.

[26] M. Kalantarnia, F. Khan, and K. Hawboldt, "Dynamic risk assessment using failure assessment and Bayesian theory," Journal of Loss Prevention in the Process Industries, vol. 22, no. 5, pp. 600-606, 2009.

[27] D. L. Kelly and C. L. Smith, "Bayesian inference in probabilistic risk assessment-the current state of the art," Reliability Engineering and System Safety, vol. 94, no. 2, pp. 628-643, 2009.

[28] "Zhangjiakou bureau of hydrology and water resources survey," Second Water Resources Assessment Report of Zhangjiakou City, Hebei Institute of Architectural Engineering, Hebei Province, 2004, (chinese).

[29] X. Wang, Q. Cui, and S. Y. Li, "An optimal water allocation model based on water resources security assessment and its application in Zhangjiakou Region, Northern China," Resources, Conservation and Recycling, vol. 69, pp. 57-65, 2012.

[30] M. A. Hamouda, M. M. Nour El-Din, and F. I. Moursy, "Vulnerability assessment of water resources systems in the Eastern Nile Basin," Water Resources Management, vol. 23, no. 13, pp. 2697-2725, 2009.

[31] P. E. Waggoner, Climate Change and US Water Resources, John Wiley \& Sons, New York, NY, USA, 1990.

[32] F. V. Jensen, An Introduction to Bayesian Networks, vol. 210, UCL Press, London, UK, 1996.

[33] J. W. K. Chan and T. K. L. Tong, "Multi-criteria material selections and end-of-life product strategy: grey relational analysis approach," Materials and Design, vol. 28, no. 5, pp. 1539-1546, 2007. 


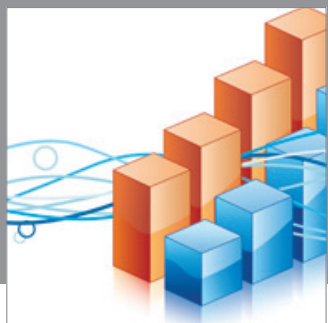

Advances in

Operations Research

mansans

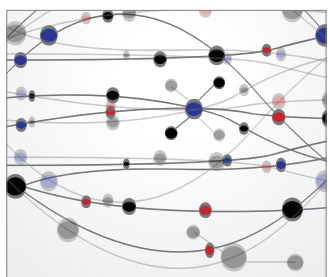

The Scientific World Journal
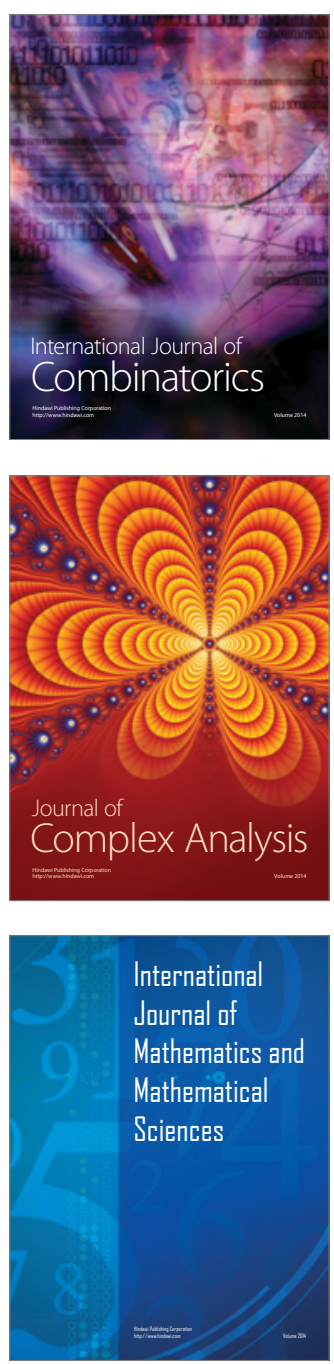
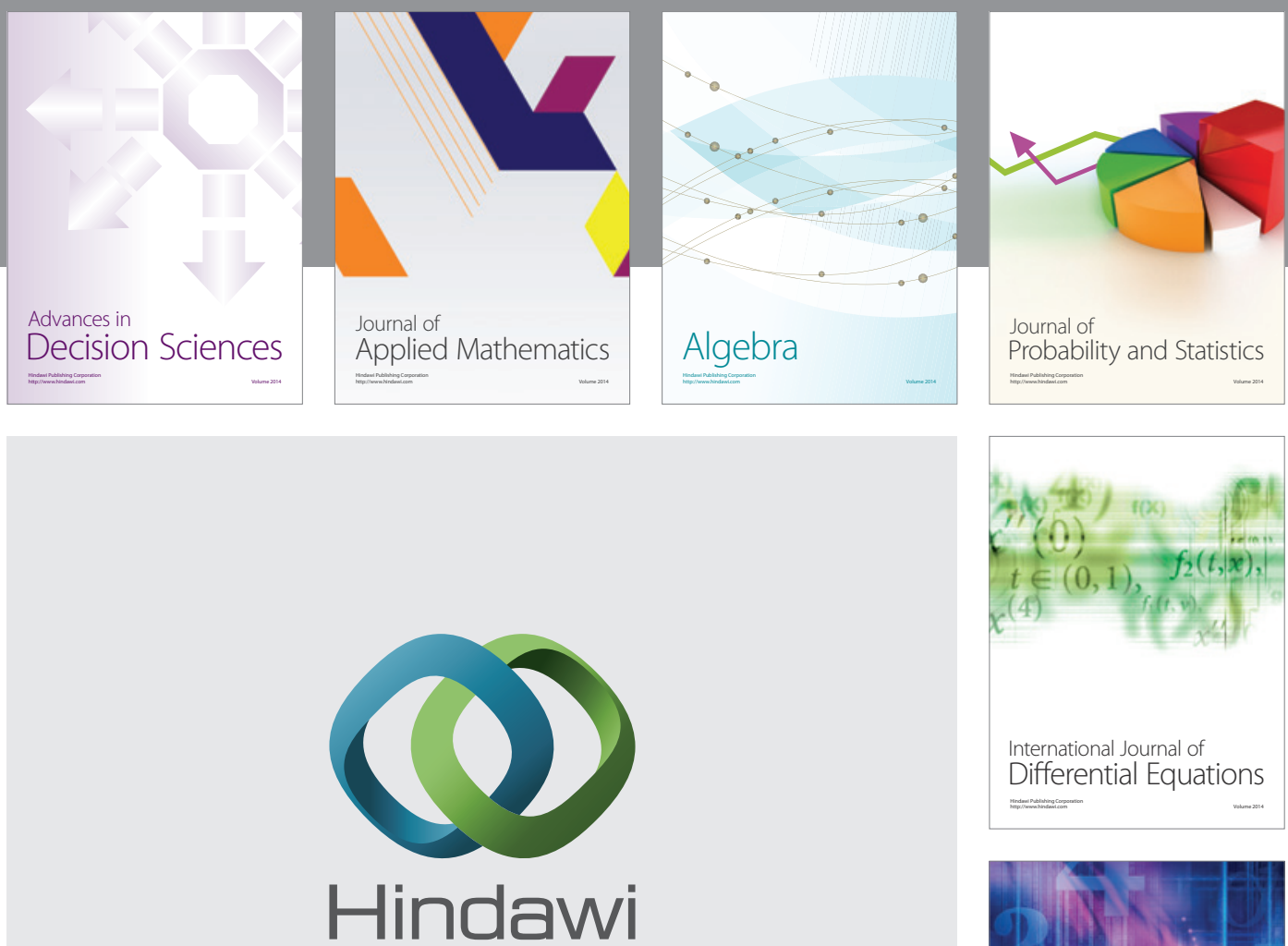

Submit your manuscripts at http://www.hindawi.com
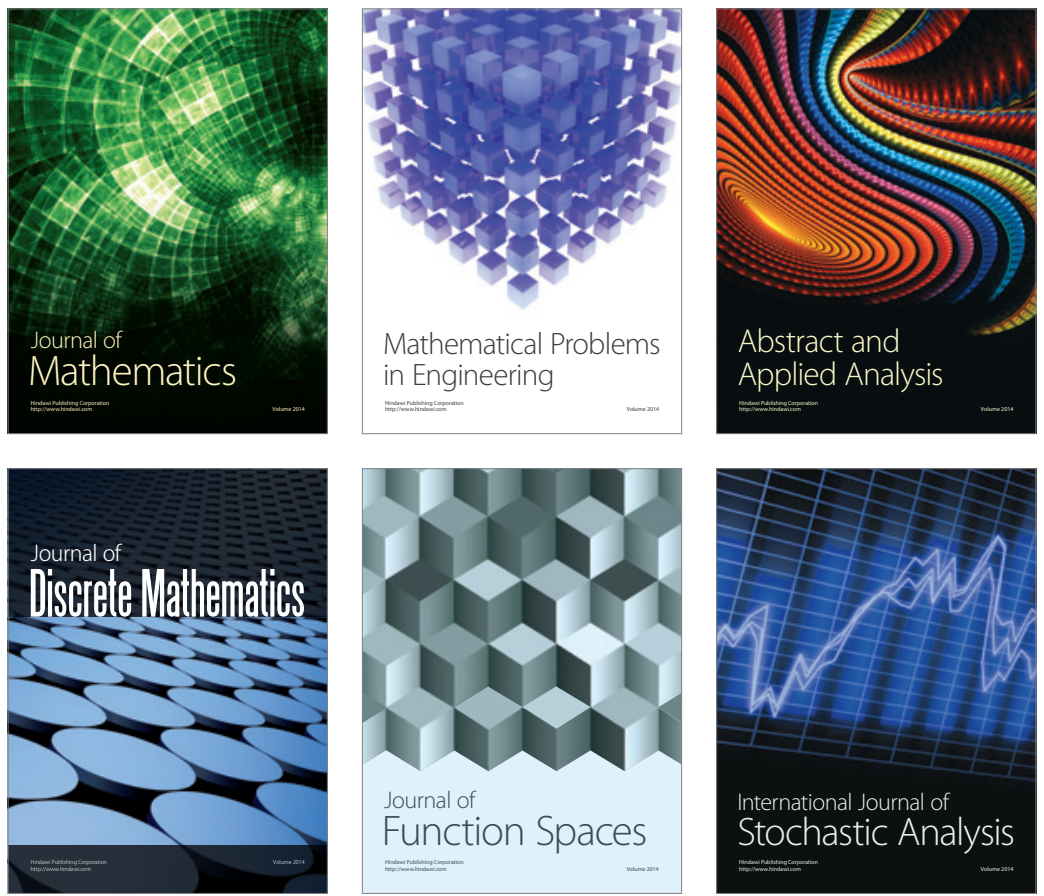

Journal of

Function Spaces

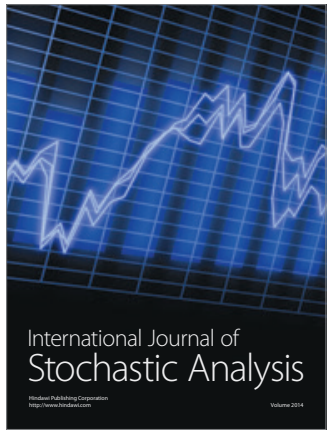

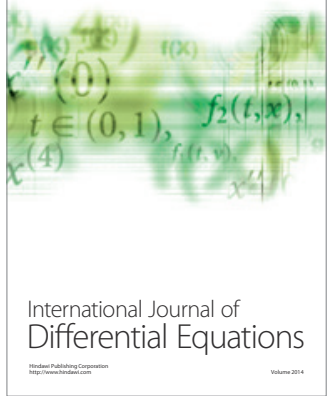
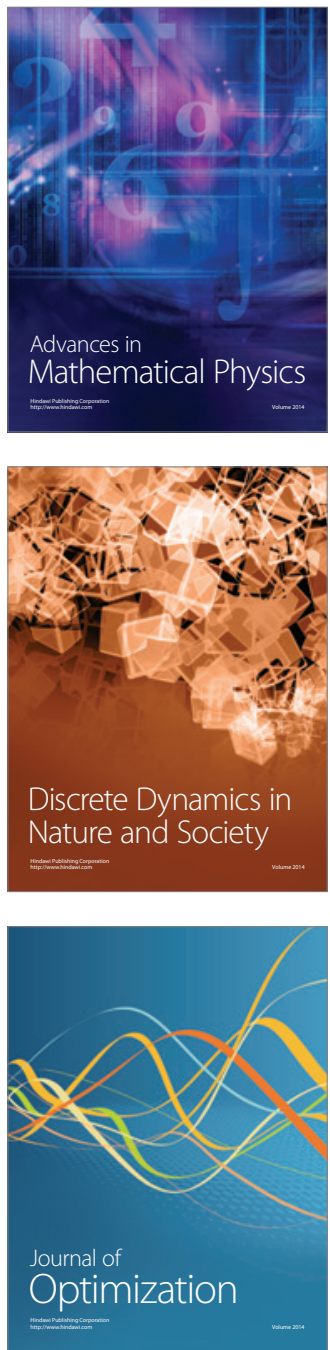